\title{
Simulations of Cyclic Voltammetry for Electric Double Layers in Asymmetric Electrolytes: A Generalized Modified Poisson-Nernst-Planck Model
}

\author{
Hainan Wang, Alexander Thiele, and Laurent Pilon* \\ Henry Samueli School of Engineering and Applied Science Mechanical and Aerospace Engineering Department, \\ University of California, Los Angeles, 420 Westwood Plaza, Los Angeles, California 90095, United States
}

\section{Supporting Information}

\begin{abstract}
This paper presents a generalized modified Poisson-Nernst-Planck (MPNP) model derived from first principles based on excess chemical potential and Langmuir activity coefficient to simulate electric double-layer dynamics in asymmetric electrolytes. The model accounts simultaneously for (1) asymmetric electrolytes with (2) multiple ion species, (3) finite ion sizes, and (4) Stern and diffuse layers along with Ohmic potential drop in the electrode. It was used to simulate cyclic voltammetry $(\mathrm{CV})$ measurements for binary asymmetric electrolytes. The results demonstrated that the current density increased significantly with decreasing ion diameter and/or increasing valency $\left|z_{i}\right|$ of either ion species. By contrast, the ion diffusion coefficients affected the CV curves and capacitance

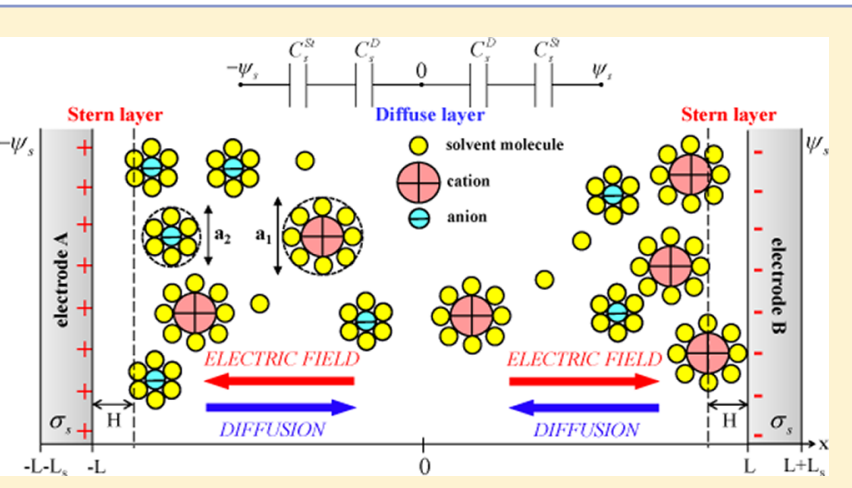
only at large scan rates. Dimensional analysis was also performed, and 11 dimensionless numbers were identified to govern the $\mathrm{CV}$ measurements of the electric double layer in binary asymmetric electrolytes between two identical planar electrodes of finite thickness. A self-similar behavior was identified for the electric double-layer integral capacitance estimated from CV measurement simulations. Two regimes were identified by comparing the half cycle period $\tau_{\mathrm{CV}}$ and the "RC time scale" $\tau_{\mathrm{RC}}$ corresponding to the characteristic time of ions' electrodiffusion. For $\tau_{\mathrm{RC}} \ll \tau_{\mathrm{CV}}$, quasi-equilibrium conditions prevailed and the capacitance was diffusion-independent while for $\tau_{\mathrm{RC}} \gg \tau_{\mathrm{CV}}$, the capacitance was diffusion-limited. The effect of the electrode was captured by the dimensionless electrode electrical conductivity representing the ratio of characteristic times associated with charge transport in the electrolyte and that in the electrode. The model developed here will be useful for simulating and designing various practical electrochemical, colloidal, and biological systems for a wide range of applications.
\end{abstract}

\section{INTRODUCTION}

Cyclic voltammetry $(\mathrm{CV})$ is a powerful technique in the field of electrochemistry. ${ }^{1-3}$ It has been the subject of intense studies in electrochemical sensing ${ }^{1-3}$ and in electrical energy storage and conversion. $^{4-9}$ In these applications, ${ }^{4-9}$ the electric potential is typically above $1 \mathrm{~V}$, while the electrolyte concentration is at least $1 \mathrm{~mol} / \mathrm{L}$. Numerous studies ${ }^{9-23}$ have demonstrated that the finite ion size must be accounted for when simulating electric double layers at such large electric potential and/or large electrolyte concentrations. However, these studies have been mostly limited to binary and symmetric electrolytes. ${ }^{9-23}$ Practical electrolytes are typically asymmetric in nature due to the difference in (i) their ion diffusion coefficients, ${ }^{24}$ (ii) their ion sizes such as in ionic liquids, ${ }^{25}$ and/or (iii) their ion valencies such as aqueous $\mathrm{H}_{2} \mathrm{SO}_{4}$ and $\mathrm{Na}_{2} \mathrm{SO}_{4}$. Moreover, the use of electrolyte mixtures with more than two ion species have been investigated in many practical applications such as supercapacitors, ${ }^{26-28}$ water desalination, ${ }^{29,30}$ electrokinetics in colloidal systems, ${ }^{31-40}$ electrochemical measurements involving supporting electrolytes, ${ }^{41-43}$ and various biological processes including gating and permeation in ion channels. ${ }^{44-50}$
Existing simulations of asymmetric electrolytes based on continuum theory and accounting for finite ion size are mainly limited to equilibrium conditions. ${ }^{21,31-40,51-62}$ For example, Bikerman $^{63}$ developed the first equilibrium modified PoissonBoltzmann (MPB) model accounting for finite ion size. This model applies to electrolytes with anions and cations having different volumes but symmetric valency. Borukhov et al. ${ }^{51,52}$ and Silalahi et al. ${ }^{53}$ developed MPB models valid for binary electrolytes with asymmetric valency but identical ion diameters. Their model was later extended to binary asymmetric electrolytes with unequal ion diameters. ${ }^{54,55}$ Biesheuvel and coworkers $^{56-58}$ and Alijó et al. ${ }^{40}$ developed more general MPB models valid for asymmetric electrolytes and/or multiple ion species with different ion sizes and valencies. This was accomplished by incorporating an excess chemical potential term based on the Boublik-Mansoori-Carnahan-Starling-Leland equation-of-state. It directly relates the excess chemical

Received: March 3, 2013

Revised: July 28, 2013

Published: August 5, 2013 
potential to the local ion concentrations, ions' effective diameters, and their exclusion volumes. ${ }^{40,56-58}$ Moreover, Tresset $^{59}$ developed a generalized Poisson-Fermi model for asymmetric electrolytes and multiple ion species based on the lattice gas approach by considering ions in discrete cells of different volumes. In addition, $\mathrm{Li}$ and co-workers ${ }^{60-62}$ developed a model for asymmetric electrolytes based on the variational principle while accounting for the finite sizes of both ions and solvent molecules. Alternatively, Horno and coworkers $^{31-37}$ developed a MPB model for asymmetric electrolytes by directly applying a "Langmuir-type" correction to the equilibrium ion concentration given by the Boltzmann distribution.

Figure 1a shows a schematic of the electric double layer structure consisting of Stern and diffuse layers forming near a

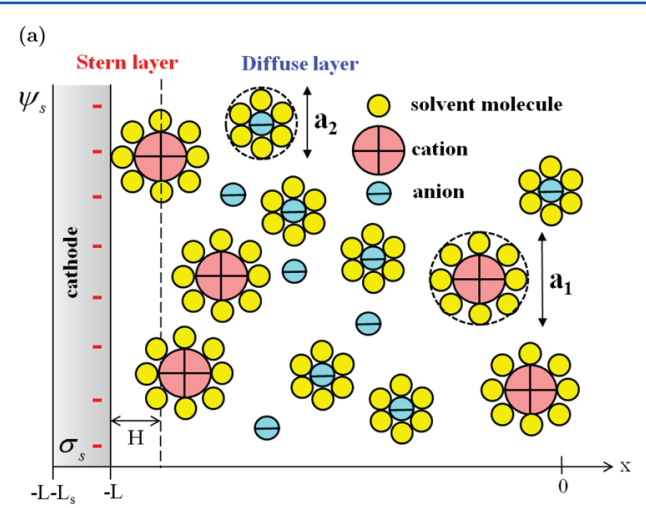

(b)

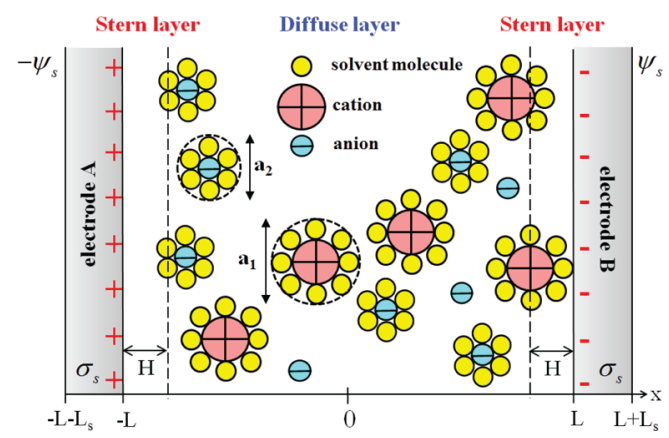

Figure 1. Schematic and the computational domain of the electric double layer structure consisting of Stern and diffuse layers (a) near a planar electrode (i.e., with the half domain) and (b) between two planar electrodes (i.e., with the full domain). Here, the ion diameters of large and small ion species are denoted by $a_{1}$ and $a_{2}$, respectively.

planar electrode. ${ }^{1,9,64}$ Several studies have considered ion species with different effective diameters resulting in multiple Stern layers with different thicknesses near the electrode surface. $^{36-40}$ There were no free charges within the Stern layer immediately adjacent to the electrode surface while all ion species coexisted in the diffuse layer. ${ }^{36-40}$ By contrast, only ion species of intermediate sizes existed in the intermediate Stern layer(s). Such electric double layer structure was typically used along with PB or MPB models. ${ }^{36-40}$ However, the associated ion concentrations do not satisfy the overall electroneutrality condition across the electrolyte domain expressed as $\sum_{i=1}^{N} \int_{-L}^{L} z_{i} c_{i}(x, t)=0$, even for uniform concentrations typically used as initial conditions. ${ }^{9,17-23}$ This was caused by the small ions present in the intermediate Stern layer(s). For example, for binary and asymmetric electrolytes with valencies $\left(z_{i}\right)_{1 \leq i \leq 2}$ and initial bulk ion concentrations $\left(c_{i \infty}\right)_{1 \leq i \leq 2}$, the above integral initially reduces to $\sum_{i=1}^{2} \int_{-L}^{L} z_{i} c_{i \infty} \mathrm{d} x=2 z_{2} c_{2 \infty}\left(H_{1}-H_{2}\right) \neq 0$ where $H_{i}=a_{i} / 2(i=1$ and 2$)$ is the Stern layer boundaries defined according to the diameters of the larger $\left(a_{1}\right)$ and smaller $\left(a_{2}\right)$ ions, respectively. Note that $c_{1}(x, t)$ and $c_{2}(x, t)$ were nonzero for $-L+H_{1} \leq x \leq L-H_{1}$ and $-L+H_{2} \leq x \leq$ $L-H_{2}$, respectively. This issue becomes particularly severe when simulating electric double layers in a finite electrolyte domain.

This paper aims to develop a model, from first principles, for simulating electric double-layer dynamics valid for asymmetric electrolytes and/or for multiple ion species accounting for different ion diameters, as well as different valencies and diffusion coefficients. The model was used to simulate $\mathrm{CV}$ measurements for binary asymmetric electrolytes.

\section{BACKGROUND}

2.1. Cyclic Voltammetry. In CV measurements, the surface potential $\psi_{\mathrm{s}}$ is imposed to vary periodically and linearly with time $t$ as

$$
\psi_{s}(t)=\left\{\begin{array}{l}
\psi_{\max }-v\left[t-2(m-1) \tau_{\mathrm{CV}}\right] \\
\quad \text { for } 2(m-1) \tau_{\mathrm{CV}} \leq t<(2 m-1) \tau_{\mathrm{CV}} \\
\psi_{\min }+v\left[t-(2 m-1) \tau_{\mathrm{CV}}\right] \\
\quad \text { for }(2 m-1) \tau_{\mathrm{CV}} \leq t<2 m \tau_{\mathrm{CV}}
\end{array}\right.
$$

where $v$ is the scan rate in $\mathrm{V} / \mathrm{s}, m(=1,2,3, \ldots)$ is the cycle number, and $\tau_{\mathrm{CV}}=\left(\psi_{\max }-\psi_{\min }\right) / v$ is half the cycle period. The latter represents the time for varying the surface potential from its maximum $\psi_{\max }$ to its minimum $\psi_{\min }$ values or versa vice. Here, $\psi_{\max }-\psi_{\min }$ is referred to as the "potential window". Note that the measurements are referred to as "linear sweep voltammetry" when $\psi_{\mathrm{s}}(t)=\psi_{\min }+v t$ or $\psi_{\mathrm{s}}(t)=$ $\psi_{\max }-v t$.

The surface charge density $q_{\mathrm{s}}$ accumulated at the electrode surface during one cycle can be estimated by computing the area enclosed by the $\mathrm{CV}$ curves. ${ }^{9}$ Then, the areal integral capacitance $C_{s}\left(\right.$ in $\left.\mathrm{F} / \mathrm{m}^{2}\right)$ can be computed from $\mathrm{CV}$ measurements as ${ }^{9}$

$$
C_{\mathrm{s}}=\frac{q_{\mathrm{s}}}{\psi_{\max }-\psi_{\min }}=\frac{1}{\psi_{\max }-\psi_{\min }} \oint \frac{j_{\mathrm{s}}}{2 v} \mathrm{~d} \psi_{\mathrm{s}}
$$

where $j_{\mathrm{s}}$ is the surface current density (in $\mathrm{A} / \mathrm{m}^{2}$ ).

2.2. Modeling of Electric Double Layer Dynamics. Recent efforts have been made to account for the effect of finite ion size in modeling ion transport in concentrated electrolyte solutions and/or under large electric potential. ${ }^{18-23,44-50,65-67}$ For example, Kilic et al. ${ }^{19}$ derived a modified Poisson-NernstPlanck (MPNP) model valid for binary and symmetric electrolytes under large electrolyte concentration and electric potential. The authors added an excess term accounting for the entropic contribution due to finite-size ions in the expression of the Helmholtz free energy. This resulted in an excess term in the expressions of the chemical potentials and mass fluxes. ${ }^{19-22}$ However, this MPNP model does not apply to asymmetric electrolytes or to multiple ion species. ${ }^{9,17-23}$

Alternatively, several authors ${ }^{65-67}$ incorporated the finite ion size in ion mass fluxes using the activity coefficient to account for the deviation from ideal electrolyte solutions. However, these studies ${ }^{65-67}$ were also limited to binary and symmetric 
electrolytes. Note that the MPNP model developed by Kilic et al. ${ }^{19}$ can be also formulated in a form equivalent to that based on the activity coefficient. ${ }^{21,22,37}$ Based on this MPNP model, ${ }^{19}$ Wang and Pilon ${ }^{9}$ performed numerical simulations reproducing $\mathrm{CV}$ measurements for electric double layer capacitors with binary and symmetric electrolytes for large concentration and potential window. The model simultaneously accounted for the finite ion size, Stern and diffuse layers, and the electrode electrical conductivity. A scaling analysis was performed, and dimensionless numbers governing $\mathrm{CV}$ measurements were identified. $^{9}$

Davidson and Goulbourne ${ }^{44}$ extended the MPNP model to multiple ion species but with symmetric ion diameter and valency. Eisenberg and co-workers ${ }^{45-47}$ developed a MPNP model for binary asymmetric electrolytes based on the variation principle. Horng et al. ${ }^{48}$ later extended this model ${ }^{45-47}$ for asymmetric electrolytes with multiple ion species. However, these MPNP models ${ }^{45-48}$ were expressed as integral-differential equations, thus making the numerical solution procedure highly involved particularly for three-dimensional geometries.

$\mathrm{Lu}$ and $\mathrm{Zhou}{ }^{49,50}$ extended the expression of chemical potential developed in refs. ${ }^{51,52}$ and proposed a "size-modified" Poisson-Nernst-Planck (SMPNP) model for asymmetric electrolytes and multiple ion species. In their model, ${ }^{49,50}$ they introduced a parameter " $k_{i}=a_{i} / a_{0}$ " representing the ratio of ion diameter $a_{i}$ and the diameter of solvent molecules $a_{0}$. The authors considered different cases for $k_{i}>1$ and justified that the model successfully constrained the ion concentrations below their maximum values. However, this SMPNP model breaks down when neglecting the size of solvent molecules and as $k_{i} \rightarrow \infty$ the excess term accounting for finite ion sizes approaches infinity.

To the best of our knowledge, no study has simulated CV curves for electric double layer capacitors with asymmetric electrolytes and/or with multiple ion species while accounting for the finite ion size. This paper aims to develop, from first principles, a model for simulating electric double-layer dynamics valid for asymmetric electrolytes and/or for multiple ion species. It simultaneously accounts for (1) asymmetric electrolytes with (2) multiple ion species of (3) finite ion size, and for (4) Stern and diffuse layers. The model will be useful for simulating electric double layers in various electrochemical, colloidal, and biological systems.

\section{ANALYSIS}

3.1. Generalized Modified Poisson-Nernst-Planck Model. Previous studies ${ }^{21,22,65-69}$ established that the effect of finite ion size on the electric double layer dynamics can be generally accounted for by employing the activity coefficient $\gamma_{i}$ in the expression of the chemical potential of each ion species " $i$ ". The ion mass flux is proportional to the gradient of chemical potential. Then, a generalized modified PoissonNernst-Planck (GMPNP) model consisting of Gauss law coupled with the mass conservation equations for each one of the $N$ ion species can be formulated as

$$
\left\{\begin{array}{c}
\nabla \cdot\left(\varepsilon_{0} \varepsilon_{\mathrm{r}} \nabla \psi\right)=-F \sum_{i=1}^{N} z_{i} c_{i} \\
\frac{\partial c_{i}}{\partial t}=\nabla \cdot\left[D_{i} c_{i}\left(\frac{F z_{i}}{R_{u} T} \nabla \psi+\nabla \ln \left(\gamma_{i} c_{i}\right)\right)\right] \\
\text { for } i=1,2, \ldots, N
\end{array}\right.
$$

where $\psi$ is the local electric potential, while $c_{i}$ and $D_{i}$ are the molar concentration and diffusion coefficient of ion species " $i$ " in the electrolyte solution, respectively. Here, $\varepsilon_{0}$ and $\varepsilon_{\mathrm{r}}$ are the free space permittivity $\left(\varepsilon_{0}=8.854 \times 10^{-12} \mathrm{~F} / \mathrm{m}\right)$ and the relative permittivity of the electrolyte solution, respectively. The temperature is denoted by $T$ (in $\mathrm{K}$ ), while $F$ and $R_{\mathrm{u}}$ are the Faraday constant $(F=96485.3 \mathrm{sA} / \mathrm{mol})$ and the universal gas constant $\left(R_{\mathrm{u}}=8.314 \mathrm{JK}^{-1} \mathrm{~mol}^{-1}\right)$, respectively.

Among various forms of activity coefficient existing in the literature, the "Langmuir type" activity coefficient ${ }^{31-37,65,70-73}$ directly relates $\gamma_{i}$ to the ion diameter $a_{i}$ to account for the exclusion volume caused by the finite size of ion species. It is expressed as ${ }^{70,71}$

$$
\gamma_{i}=\frac{1}{1-\sum_{i=1}^{N} \frac{c_{i}}{c_{i, \max }}}
$$

where $c_{i, \max }$ is the maximum ion concentration of ion species " $i$ " and is given by $c_{i, \max }=1 / N_{\mathrm{A}} a_{i}^{3}$ when assuming simple cubic ion packing. Here, $N_{\mathrm{A}}$ is the Avogadro's number $\left(N_{\mathrm{A}}=6.022 \times\right.$ $\left.10^{23} \mathrm{~mol}^{-1}\right)$. Note that as the ion diameter vanishes, i.e., $a_{i}=0$, $c_{i, \max }$ tends to infinity and $\gamma_{i}$ approaches 1 . It is also interesting to note that eq 4 is a simplified expression of the activity coefficient based on the Boublik-Mansoori-CarnahanStarling-Leland equation-of-state. ${ }^{21,56-58}$

By employing the "Langmuir type" activity coefficient given by eq 4 , the GMPNP model can be written as

$$
\left\{\begin{array}{l}
\nabla \cdot\left(\varepsilon_{0} \varepsilon_{\mathrm{r}} \nabla \psi\right)=-F \sum_{i=1}^{N} z_{i} c_{i} \\
\frac{\partial c_{i}}{\partial t}=\nabla \cdot\left(\frac{D_{i} F z_{i} c_{i}}{R_{\mathrm{u}} T} \nabla \psi+D_{i} \nabla c_{i}+\frac{D_{i} N_{\mathrm{A}} c_{i} \sum_{i=1}^{N} a_{i}^{3} \nabla c_{i}}{1-N_{\mathrm{A}} \sum_{i=1}^{N} a_{i}^{3} c_{i}}\right)
\end{array}\right.
$$

Note that for vanishing ion diameter $\left(a_{i}=0\right)$, eqs 3 and 5 reduce to the classical PNP model. ${ }^{9}$ In addition, for binary and symmetric electrolytes, $N=2, z_{1}=-z_{2}, D_{1}=D_{2}$, and $a_{1}=a_{2}$, and eqs 3 and 5 reduce to the MPNP model developed in refs 19 and 21. Note also that the GMPNP model can be also derived by considering the excess chemical potential $\mu_{i}^{\text {ex }}$ for each ion species " $i$ " and by noting its direct relation to the activity coefficient $\gamma_{i}$ given by $\mu_{i}^{\mathrm{ex}}=k_{\mathrm{B}} T \ln \left(\gamma_{i}\right) .^{21,68,70}$

Compared with previous MPNP models, ${ }^{9,17-23,44-50,65-67}$ the present generalized MPNP (GMPNP) model, given by eqs 3 to 5 , has several attractive features. First, it applies to asymmetric electrolytes and/or multiple ion species. Second, it does not present any additional challenges in the numerical solution procedure compared with the MPNP model for binary symmetric electrolytes. ${ }^{9,17,19-23}$ Moreover, it can be conveniently applied to three-dimensional geometries unlike those expressed as integral-differential equations. ${ }^{45-48}$ Third, it is consistent with the classical or existing models when considering ions as point charges or having identical diameters. Fourth, it can be readily extended by employing other forms of activity coefficient based on available expressions of excess chemical potential $\mu_{i}^{\text {ex }}$ such as the Boublik-MansooriCarnahan-Starling-Leland equation-of-state. ${ }^{21,58}$

3.2. Schematics and Assumptions. In the present study, the double layer structure with multiple Stern layers proposed in refs 36-40 was not adopted because this does not satisfy the overall electroneutrality condition, as discussed previously. Instead, the electric double layer was assumed to consist of (i) a single Stern layer adjacent to the electrode surface and (ii) a diffuse layer beyond. Wang et al., ${ }^{916}$ previously showed that the 
electrode curvature has negligible effect on the areal integral capacitance for electrode or pore radii larger than $40 \mathrm{~nm}$. Therefore, analysis of planar electrodes is representative of macroporous and mesoporous electrodes with large enough radii of curvature.

Figure 1a shows the half domain used in previous simulations for binary and symmetric electrolytes. ${ }^{9}$ By contrast, Figure $1 \mathrm{~b}$ shows the schematic of the computational domain used to simulate the electrolyte solution consisting of binary asymmetric electrolytes between two identical planar electrodes A and $\mathrm{B}$. In the present study, simulations with the former and the latter domains were referred to as "half-domain simulations" and "full-domain simulations", respectively. Here, the effective ion diameters of the larger and smaller ion species were denoted by $a_{1}$ and $a_{2}$, respectively. The region of electrolyte solution consisted of three layers including (1) the Stern layer of thickness $H$ near each electrode surface located at $x= \pm L$ and (2) the diffuse layer between the Stern layers, i.e., $-L+$ $H \leq x \leq L-H$. Here, the thickness $H$ was approximated as the effective radius of the largest ion referred to as ion species 1, i.e., $H=a_{1} / 2$.

To make the problem mathematically tractable, the following assumptions were made: (1) the effective ion diameter was assumed to be independent of electrolyte concentration, ${ }^{19,21,74}$ (2) the electrolyte dielectric permittivity was constant and equal to that of water. Note that the dielectric permittivity may depend on electric field and temperature. ${ }^{13,16,75,76}$ However, it is typically assumed to be constant in scaling analysis, as performed in refs 11, 12, 19-23, and 77. (3) isothermal conditions prevailed throughout the electrode and electrolyte, (4) advection of the electrolyte was assumed to be negligible, (5) the ions could only accumulate at the electrode surface and could not diffuse into the electrode, i.e., there was no ion insertion, and (6) the specific ion adsorption due to nonelectrostatic forces was assumed to be negligible.

3.3. One-Dimensional Formulation. The local electric potential $\psi(x, t)$ in the identical solid electrodes $\mathrm{A}$ and $\mathrm{B}$ of electrical conductivity $\sigma_{\mathrm{s}}$ and thickness $L_{\mathrm{s}}$ was governed by the Poisson equation expressed as ${ }^{9,78,79}$

$$
\frac{\partial}{\partial x}\left(\sigma_{\mathrm{s}} \frac{\partial \psi}{\partial x}\right)=0 \text { for }-L_{\mathrm{s}}-L \leq x<-L \text { and } L<x \leq L+L_{\mathrm{s}}
$$

Moreover, the local electric potential $\psi(x, t)$ and molar ion concentrations $c_{i}(x, t)$ at time $t$ and location $x$ in the binary asymmetric electrolyte solution were computed by solving the generalized MPNP model with a Stern layer. For planar electrodes, the generalized MPNP model (eqs 5) in the diffuse layer $(-L+H \leq x \leq L-H)$ can be expressed in its onedimensional form as

$$
\left\{\begin{array}{l}
\frac{\partial}{\partial x}\left(\varepsilon_{0} \varepsilon_{\mathrm{r}} \frac{\partial \psi}{\partial x}\right)=-F \sum_{i=1}^{2} z_{i} c_{i} \\
\frac{\partial c_{i}}{\partial t}=-\frac{\partial N_{i}}{\partial x}
\end{array}\right.
$$

Here, $N_{i}$ denotes the mass flux of ion species “ $i$ ” expressed as

$$
\begin{gathered}
N_{i}(x, t)=-\frac{D_{i} F z_{i} c_{i}}{R_{\mathrm{u}} T} \frac{\partial \psi}{\partial x}-D_{i} \frac{\partial c_{i}}{\partial x} \\
-\frac{D_{i} N_{\mathrm{A}} c_{i}}{1-N_{\mathrm{A}} \sum_{i=1}^{2} a_{i}^{3} c_{i}} \frac{\partial}{\partial x}\left(\sum_{i=1}^{2} a_{i}^{3} c_{i}\right)
\end{gathered}
$$

Here, the surface potentials at the surface of electrode $\mathrm{A}(x=$ $\left.-L-L_{\mathrm{s}}\right)$ and $\mathrm{B}\left(x=L+L_{\mathrm{s}}\right)$ were imposed to be $-\psi_{\mathrm{s}}(t)$ and $+\psi_{s}(t)$, respectively, where $\psi_{s}(t)$ is given by eq 1 . Moreover, the Stern layers can be accounted for via the boundary conditions relating the potential drop across the Stern layer and the potential gradient at the Stern/diffuse layer interface located at $x=(L-H)$ and $x=-(L-H){ }^{16,19,21}$ Then, it suffices to simulate only the diffuse layer in the computational domain defined by $-L+H \leq x \leq L-H .^{16,19,21}$ For planar electrodes, the boundary conditions accounting for the Stern layers are given by ${ }^{16,19,21}$

$$
\begin{gathered}
\frac{\partial \psi}{\partial x}(x=L-H, t)=\frac{1}{H}[\psi(x=L, t) \\
-\psi(x=L-H, t)]
\end{gathered}
$$

and

$$
\begin{gathered}
-\frac{\partial \psi}{\partial x}(x=-L+H, t)=\frac{1}{H}[\psi(x=-L, t) \\
-\psi(x=-L+H, t)]
\end{gathered}
$$

The electric potential and current density were continuous across the electrode/electrolyte interface located at $x= \pm L$ so that $^{9,80}$

$$
\begin{aligned}
& \psi\left(x=-L^{-}, t\right)=\psi\left(x=-L^{+}, t\right) \quad \text { and } \\
& -\sigma_{\mathrm{s}} \frac{\partial \psi}{\partial x}\left(x=-L^{-}, t\right)=-\varepsilon_{0} \varepsilon_{\mathrm{r}} \frac{\partial^{2} \psi}{\partial x \partial t}\left(x=-L^{+}, t\right) \\
& \psi\left(x=L^{-}, t\right)=\psi\left(x=L^{+}, t\right) \quad \text { and } \\
& \quad-\sigma_{\mathrm{s}} \frac{\partial \psi}{\partial x}\left(x=L^{+}, t\right)=-\varepsilon_{0} \varepsilon_{\mathrm{r}} \frac{\partial^{2} \psi}{\partial x \partial t}\left(x=L^{-}, t\right)
\end{aligned}
$$

Moreover, at the Stern/diffuse layer interface located at $x= \pm$ $(L-H)$, the mass fluxes of ion species vanish since there is no ion insertion in the electrode material (assumption 5) such that

$$
N_{i}(-L+H, t)=0 \quad \text { and } \quad N_{i}(L-H, t)=0
$$

The initial conditions in the diffuse layer satisfy the electroneutrality condition and are given by

$$
\begin{gathered}
\psi(x, t=0)=0, c_{1}(x, t=0)=c_{1, \infty} \text { and } \\
c_{2}(x, t=0)=-c_{1, \infty} z_{1} / z_{2}
\end{gathered}
$$

where $c_{i, \infty}$ represents the bulk ion concentration of ion species " $i$ ".

Note that the Ohmic IR drop due to the electrolyte solution is typically the major source of resistance. It is often accounted for via a resistance in an equivalent $\mathrm{RC}$ circuit while ignoring the electric double layer structure. ${ }^{1,81}$ By contrast, the present study accounted for the detailed electric double layer structure. Thus, the electric potential drop in the electrolyte solution was rigorously obtained by numerically solving the GMPNP model along with the boundary conditions (eqs 7-12).

3.4. Dimensional Analysis. Equations 6-12 constitute the one-dimensional generalized MPNP model with a Stern layer 
for binary asymmetric electrolytes accounting for the electrodes. Based on our previous studies, ${ }^{9}$ the following scaling parameters were introduced:

$$
x^{*}=\frac{x}{\lambda_{D}}, t^{*}=\frac{t D_{1}}{\lambda_{D}^{2}}, \psi^{*}=\frac{\psi}{R_{\mathrm{u}} T / z_{1} F}, \text { and } c_{i}^{*}=\frac{c_{i}}{c_{1, \infty}}
$$

where $\lambda_{\mathrm{D}}=\left(\varepsilon_{0} \varepsilon_{\mathrm{r}} R_{\mathrm{u}} T / F^{2} \sum_{i=1}^{2} z_{i}^{2} c_{i, \infty}\right)$ is the Debye length for binary asymmetric electrolytes. ${ }^{82}$ Then, the governing eq 6 in the electrode can be expressed, in dimensionless form, as

$$
\begin{gathered}
\frac{\partial}{\partial x^{*}}\left(\frac{\partial \psi^{*}}{\partial x^{*}}\right)=0 \text { for }-\left(L_{\mathrm{s}}+L\right) / \lambda_{D} \leq x<-L / \lambda_{D} \\
\text { and } L / \lambda_{D}<x \leq\left(L+L_{\mathrm{s}}\right) / \lambda_{D}
\end{gathered}
$$

Similarly, the governing eqs 7 in the electrolyte were transformed into the dimensionless as,

$$
\left\{\begin{array}{c}
\frac{\partial^{2} \psi^{*}}{\partial x^{* 2}}=-\frac{c_{1}^{*}+z_{2}^{*} c_{2}^{*}}{1-z_{2}^{*}} \\
\frac{\partial c_{i}^{*}}{\partial t^{*}}=-\frac{\partial N_{i}^{*}}{\partial x^{*}}
\end{array}\right.
$$

for $\left(-L+a_{1} / 2\right) / \lambda_{\mathrm{D}} \leq x^{*} \leq\left(L-a_{1} / 2\right) / \lambda_{\mathrm{D}}$ with the dimensionless ion flux $\mathrm{N}_{i}^{*}$ given by

$$
\begin{aligned}
N_{i}^{*}\left(x^{*}, t^{*}\right)= & -\frac{D_{i}}{D_{1}} \frac{\partial c_{i}^{*}}{\partial x^{*}}-\frac{D_{i}}{D_{1}} \frac{z_{i}}{z_{1}} c_{i}^{*} \frac{\partial \psi^{*}}{\partial x^{*}} \\
& -\frac{D_{i}}{D_{1}} \frac{c_{i}^{*}}{2-\sum_{i=1}^{2} \nu_{\mathrm{p} i} c_{i}^{*}} \frac{\partial}{\partial x^{*}} \sum_{i=1}^{2} \nu_{\mathrm{p} i} c_{i}^{*}
\end{aligned}
$$

where $z_{2}^{*}=z_{2} / z_{1}$ is the ratio of valencies between smaller and larger ion species, i.e., $z_{2}^{*}=-1$ for symmetric electrolytes. Here, the packing parameter for ion species " $i$ " is defined as $v_{p i}=2 c_{1 \infty} /\left(1 / a_{i}^{3} N_{\mathrm{A}}\right)$. It represents the ratio of the total bulk ion concentration to the maximum ion concentration $1 / a_{i}^{3} N_{\mathrm{A}}$ assuming a simple cubic packing of ions of diameter $a_{i}$.

The dimensionless potential $\psi_{s}^{*}(t)$ imposed at the electrode surface in $\mathrm{CV}$ measurements is given by

$$
\psi_{\mathrm{s}}^{*}\left(t^{*}\right)=\left\{\begin{array}{c}
\psi_{\max }^{*}-v^{*}\left[t^{*}-2(m-1) \tau_{\mathrm{CV}}{ }^{*}\right] \\
\text { for } 2(m-1) \tau_{\mathrm{CV}}{ }^{*} \leq t^{*} \leq(2 m-1) \tau_{\mathrm{CV}} \\
\psi_{\min }^{*}+v^{*}\left[t^{*}-(2 m-1) \tau_{\mathrm{CV}}{ }^{*}\right] \\
\text { for }(2 m-1) \tau_{\mathrm{CV}}{ }^{*} \leq t^{*} \leq 2 m \tau_{\mathrm{CV}}{ }^{*}
\end{array}\right.
$$

where $\tau_{\mathrm{CV}}^{*}=\left(\psi_{\max }^{*}-\psi_{\min }^{*}\right) / v^{*}$ represents the dimensionless half cycle period and $v^{*}=\left(\lambda_{D}^{2} / D_{1}\right) /\left[\left(R_{\mathrm{u}} T / z_{1} F\right) / v\right]$ is the dimensionless scan rate. It can be interpreted as the ratio of the ion diffusion time scale $\left(\lambda_{D}^{2} / D_{1}\right)$ and the characteristic time for reaching the thermal potential $R_{\mathrm{u}} T / z_{1} F$ at scan rate $v$. Moreover, $\psi_{\max }^{*}=\psi_{\max } /\left(R_{\mathrm{u}} T / z_{1} F\right)$ and $\psi_{\min }^{*}=\psi_{\min } /\left(R_{\mathrm{u}} T / z_{1} F\right)$ are the maximum and minimum surface potentials, respectively, scaled by the thermal potential. They can be also interpreted as the ratio of characteristic times to reach $\psi_{\max }$ or $\psi_{\min }$ and the characteristic time for reaching the thermal potential at scan rate $v$.

Moreover, the boundary conditions [eqs 9 and 11] at the Stern/diffuse layer interface located at $x^{*}= \pm\left(L-a_{1} / 2\right) / \lambda_{\mathrm{D}}$ can be written in dimensionless form as

$$
\begin{aligned}
& \frac{2}{a_{1}^{*}} \frac{\partial \psi^{*}}{\partial x^{*}}\left(x^{*}=L^{*}-a_{1}^{*} / 2, t^{*}\right) \\
& \quad=\psi^{*}\left(x^{*}=L^{*}, t^{*}\right)-\psi^{*}\left(x^{*}=L^{*}-a_{1}^{*} / 2, t^{*}\right) \\
& -\frac{2}{a_{1}^{*}} \frac{\partial \psi^{*}}{\partial x^{*}}\left(x^{*}=-L^{*}+a_{1}^{*} / 2, t^{*}\right) \\
& \quad=-\psi^{*}\left(x^{*}=-L^{*}, t^{*}\right)-\psi^{*}\left(x^{*}=-L^{*}+a_{1}^{*} / 2, t^{*}\right)
\end{aligned}
$$

$$
N_{i}^{*}\left(L^{*}-a_{1}^{*} / 2, t^{*}\right)=N_{i}^{*}\left(-L^{*}+a_{1}^{*} / 2, t^{*}\right)=0
$$

where $L^{*}=L / \lambda_{\mathrm{D}}$ and $a_{1}^{*}=a_{1} / \lambda_{\mathrm{D}}$ are respectively the half interelectrode distance and effective ion diameter scaled by the Debye length representing the thickness of the electric double layer. Similarly, the boundary conditions (eqs 10) at the electrode/electrolyte interface located at $x^{*}= \pm L / \lambda_{\mathrm{D}}$ became, in dimensionless form,

$$
\psi^{*}\left(x^{*}= \pm L^{ \pm} / \lambda_{D}\right)=\psi^{*}\left(x^{*}= \pm L^{\mp} / \lambda_{D}\right)
$$

and

$$
\begin{gathered}
\frac{\sigma_{\mathrm{s}}^{*}}{\psi_{\max }^{*}-\psi_{\min }^{*}} \frac{L_{\mathrm{s}}^{*}}{L^{*}} \frac{\partial \psi^{*}}{\partial x^{*}}\left(x^{*}= \pm L^{ \pm} / \lambda_{D}\right) \\
=\frac{\partial^{2} \psi^{*}}{\partial x^{*} \partial t^{*}}\left(x^{*}= \pm L^{\mp} / \lambda_{D}\right)
\end{gathered}
$$

where $L_{\mathrm{s}}^{*}=L_{\mathrm{s}} / \lambda_{\mathrm{D}}$ is the electrode thickness scaled by the Debye length. Note that $\sigma_{\mathrm{s}}^{*}=\left[\sigma_{\mathrm{s}}\left(\psi_{\max }-\psi_{\min }\right) / L_{\mathrm{s}}\left(F z_{1} c_{1 \infty} D_{1} / L\right)\right]$ represents the ratio of the characteristic current density in the electrode to that in the electrolyte. It can be also interpreted as the ratio of time scales for charge transport in the electrolyte and in the electrode.

Similarly, the dimensionless initial conditions for $\psi^{*}$ and $c_{i}^{*}$ in the diffuse layer, for $-L^{*}+a_{1}^{*} / 2 \leq x^{*} \leq L^{*}-a_{1}^{*} / 2$, simplify as

$$
\begin{aligned}
& \psi^{*}\left(x^{*}, t^{*}=0\right)=0, \quad c_{1}^{*}\left(x^{*}, t^{*}=0\right)=1, \quad \text { and } \\
& c_{2} *\left(x^{*}, t^{*}=0\right)=-1 / z_{2}{ }^{*}
\end{aligned}
$$

Considering the dimensionless governing equations and associated boundary and initial conditions, eleven key dimensionless similarity parameters can be identified as

$$
\begin{aligned}
& v^{*}=\frac{\lambda_{\mathrm{D}}^{2} / D_{1}}{\left(R_{\mathrm{u}} T / z_{1} F\right) / v}, \psi_{\max }^{*}=\frac{\psi_{\max }}{R_{\mathrm{u}} T / z_{1} F}, \\
& \psi_{\min }^{*}=\frac{\psi_{\min }}{R_{\mathrm{u}} T / z_{1} F}, L^{*}=\frac{L}{\lambda_{D}}, a_{1}^{*}=\frac{a_{1}}{\lambda_{D}} \\
& \nu_{\mathrm{p} 1}=2 a_{1}^{3} N_{\mathrm{A}} c_{1 \infty}, \quad \nu_{\mathrm{p} 2}=2 a_{2}^{3} N_{\mathrm{A}} c_{1 \infty}, D_{2}^{*}=\frac{D_{2}}{D_{1}}, \\
& z_{2}^{*}=\frac{z_{2}}{z_{1}}, \sigma_{\mathrm{s}}^{*}=\frac{\sigma_{s}\left(\psi_{\max }-\psi_{\min }\right) / L_{\mathrm{s}}}{F z_{1} c_{1 \infty} D_{1} / L}, \text { and } L^{*}=\frac{L_{\mathrm{s}}}{\lambda_{D}}
\end{aligned}
$$

where $v_{\mathrm{p} 1}$ represents the packing parameter due to finite ion size of ion species " 1 ". Note that $v^{*}, \psi_{\max }^{*}, \psi_{\min }^{*}, L^{*}, a_{1}^{*}$, and $v_{\mathrm{p} 1}$ were identical to or direct functions of those identified in ref 9 for the CV simulations of electric double layer capacitors with binary symmetric electrolytes. When considering binary asymmetric electrolytes, three additional dimensionless 
numbers appear, namely, $v_{\mathrm{p} 2}, D_{2}^{*}$, and $z_{2}^{*}$. The dimensionless numbers $\sigma_{\mathrm{s}}^{*}$ and $L_{\mathrm{s}}^{*}$ account for the charge transport in the electrode.

3.5. Constitutive Relations. In order to solve eqs 7-12 or eqs 15-20, the electrolyte properties $\varepsilon_{\mathrm{r}}, a_{i}, z_{i}, D_{i}, c_{1 \infty}$ along with the temperature $T$, and the surface potential $\psi_{s}(t)$ are needed. The present study focuses on binary electrolytes at room temperature $T=298 \mathrm{~K}$ with different ion diameter, diffusion coefficient, and/or valency. The electrolyte relative permittivity was taken as that of water $\varepsilon_{\mathrm{r}}=78.5,{ }^{24}$ while the effective ion diameter $a_{i}$ ranged from 0.60 to $1.0 \mathrm{~nm}$ typical of solvated ion diameters. ${ }^{74}$ The valencies $\left(z_{1}: z_{2}\right)$ corresponded also to realistic conditions including $1:-1,1:-2,2:-2$, or $1:-3$. The ion diffusion coefficients $D_{i}$ varied from $1.957 \times 10^{-9} \mathrm{~m}^{2} / \mathrm{s}$ to $5.273 \times 10^{-9} \mathrm{~m}^{2} / \mathrm{s}$ representative of aqueous and organic electrolytes. $^{24}$ The initial and bulk ion concentrations was $c_{1, \infty}=1.0 \mathrm{~mol} / \mathrm{L}$ and $c_{2}=-c_{1, \infty} z_{1} / z_{2}$ satisfying the overall electroneutrality condition. Moreover, the maximum and minimum surface electric potentials were $\psi_{\max }=0.5 \mathrm{~V}$ and $\psi_{\text {min }}=-0.5 \mathrm{~V}$, respectively. The scan rate in actual $\mathrm{CV}$ measurements for electrical energy storage devices ranges typically from $10^{-3}$ to $200 \mathrm{~V} / \mathrm{s}^{4-8}$ Here, the scan rate $v$ varied over a wider range from $10^{-2}$ to $10^{8} \mathrm{~V} / \mathrm{s}$. This was due to two main reasons. First, small electrode thickness $(0-100 \mathrm{~nm})$ was considered along with realistic values $(>1 \mu \mathrm{m})$ to validate the scaling analysis. The capacitance starts to decrease at very large scan rate for such small electrode thickness. ${ }^{9}$ Second, asymptotic behaviors of electric double layers were explored at very large scan rates. Our previous study ${ }^{9}$ established that electric double layers behave as a resistor under such conditions. Finally, the electrode electrical conductivity was chosen to be $10^{-5}$ to $10^{2} \mathrm{~S} / \mathrm{m}$, typical of carbon materials. ${ }^{83,84}$

3.6. Method of Solution. The governing eqs 7 and 8 were solved along with the boundary and initial conditions given by eqs 9-12 using the commercial finite element solver COMSOL 4.2. CV measurements were simulated by numerically imposing the periodic surface electric potential given by eq 1 .

Simulations of $\mathrm{CV}$ measurements were performed for at least three cycles. The numerical convergence criterion was defined such that the maximum relative difference in the predicted capacitive current density $j_{C}$ was less than $1 \%$ when (i) reducing the mesh size by a factor of 2 and (ii) dividing the time step by two. The mesh size was the smallest at the electrode surfaces due to the large potential gradient and then gradually increased. Converged solutions were achieved by imposing a time step of $\Delta t \approx t_{0} / 800=\left(\psi_{\max }-\psi_{\min }\right) / 800 v$ with the mesh size $\Delta x / L=$ $5 \times 10^{-14}$ at the Stern/diffuse layer interface with a growth rate of 1.3 up to $\Delta x / L=1 / 250$ in the rest of the domain. Based on these convergence criteria, the total number of finite elements was less than 1910 for all cases simulated in the present study.

3.7. Data Processing. The capacitive current density $j_{C}$ from CV simulations was computed based on its definition as $^{9,85-88}$

$$
\begin{aligned}
j_{\mathrm{C}}(t)= & -\left.\varepsilon_{0} \varepsilon_{\mathrm{r}} \frac{\partial^{2} \psi}{\partial x \partial t}\right|_{x_{L} \text { or }-x_{L}} \text { at } x_{L}=L-H \text { or } \\
& -x_{L}=-(L-H)
\end{aligned}
$$

The corresponding dimensionless capacitive current density $j_{\mathrm{C}}^{*}$ at $x_{L}^{*}=L^{*}-a_{1}^{*} / 2$ and $-x_{L}^{*}=-L^{*}+a_{1}^{*} / 2$ is expressed as

$$
j_{\mathrm{C}}^{*}\left(t^{*}\right)=\frac{j_{\mathrm{C}}(t)}{F z_{1} D_{1} c_{1, \infty} / \lambda_{D}}=-\left.\sum_{i=1}^{2} z_{i}^{*^{2}} c_{i, \infty}^{*} \frac{\partial^{2} \psi^{*}}{\partial x^{*} \partial t^{*}}\right|_{x_{L}^{*} \text { or }-x_{L}^{*}}
$$

In order to reproduce typical $\mathrm{CV}$ measurements, the current densities $j_{\mathrm{C}}(t)$ and $j_{\mathrm{C}}^{*}\left(t^{*}\right)$ were plotted as functions of $\psi_{s}(t)$ and $\psi_{\mathrm{s}}^{*}\left(t^{*}\right)$, respectively.

3.8. Validation. The numerical tool was validated based on existing solutions reported in the literature. The transient ion concentration and electric potential profiles were predicted by solving the PNP and MPNP models for binary and symmetric electrolytes $\left(N=2, z_{1}=-z_{2}=z\right.$, and $\left.a_{1}=a_{2}\right)$ with constant surface electric potential. The results were successfully compared with the reported numerical solutions for $c_{i}(x, t)$ and $\psi(x, t)$ for a wide range of packing parameter $v_{\mathrm{p}}$ and dimensionless surface potential $\psi_{\mathrm{s}}^{*}$. ${ }^{\text {. }}$

\section{RESULTS AND DISCUSSION}

Results in the Supporting Information (Figures S2 and S3) demonstrate that half-domain CV simulations should only be used for symmetric electrolytes while the full domain must be simulated for asymmetric electrolytes. Consequently, all the following CV simulations were performed for the entire domain consisting of electrolyte solution between two identical planar electrodes.

4.1. Asymmetric versus Symmetric Electrolytes. 4.1.1. Effect of Asymmetric Ion Diameter. Figure 2 shows

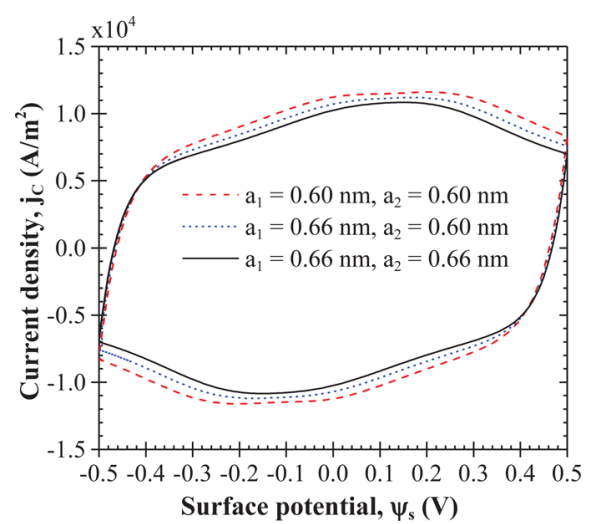

Figure 2. Predicted $j_{\mathrm{C}}$ versus $\psi_{\mathrm{s}}$ curves from CV simulations for three cases with different ion diameters, namely, (i) $a_{1}=a_{2}=0.60 \mathrm{~nm}$, (ii) $a_{1}=0.66$ and $a_{2}=0.60 \mathrm{~nm}$, and (iii) $a_{1}=a_{2}=0.66 \mathrm{~nm}$. Results were obtained by solving the GMPNP model with a Stern layer (eqs 6-12) without accounting for the potential drop across the electrodes corresponding to $\sigma_{s} \rightarrow \infty \mathrm{S} / \mathrm{m}$ or $L_{s}=0 \mathrm{~m}$. Other parameters were $c_{1, \infty}=1 \mathrm{~mol} / \mathrm{L}, v=10^{4} \mathrm{~V} / \mathrm{s}, L=200 \mu \mathrm{m}, \psi_{\max }=\psi_{\min }=0.5 \mathrm{~V}$, $T=298 \mathrm{~K}$, and $\varepsilon_{\mathrm{r}}=78.5$.

CV curves predicted for three cases with different ion diameters, namely (i) $a_{1}=a_{2}=0.60 \mathrm{~nm}$, (ii) $a_{1}=0.66 \mathrm{~nm}$ and $a_{2}=0.60 \mathrm{~nm}$, and (iii) $a_{1}=a_{2}=0.66 \mathrm{~nm}$. The ion valencies and diffusion coefficients were $z_{1}=-z_{2}=1$ and $D_{1}=D_{2}=$ $1.957 \times 10^{-9} \mathrm{~m}^{2} / \mathrm{s}$, respectively. Other parameters were $c_{1, \infty}=$ $1 \mathrm{~mol} / \mathrm{L}, v=10^{4} \mathrm{~V} / \mathrm{s}, L=200 \mu \mathrm{m}, \psi_{\max }=\psi_{\min }=0.5 \mathrm{~V}$, $T=298 \mathrm{~K}$, and $\varepsilon_{\mathrm{r}}=78.5$. Results were obtained by solving the GMPNP model with a Stern layer (eqs 6-12) without accounting for the potential drop across the electrodes corresponding to $\sigma_{\mathrm{s}} \rightarrow \infty \mathrm{S} / \mathrm{m}$ or $L_{\mathrm{s}}=0 \mathrm{~m}$. Figure $2 \mathrm{dem}$ onstrates that the current density $j_{\mathrm{C}}$ for asymmetric electrolyte with $a_{1}=0.66 \mathrm{~nm}$ and $a_{2}=0.60 \mathrm{~nm}$ lay between those obtained 
for symmetric electrolytes with ion diameter $a$ equal to 0.66 and $0.60 \mathrm{~nm}$. The relative difference in $j_{C}$ between asymmetric and symmetric electrolytes was about 9\%. Figure 2 also indicates that the predicted current density $j_{C}$ increased with decreasing ion diameter. This can be attributed to the fact that smaller ions have larger maximum ion concentrations $c_{i \text { max }}$ and thus feature a larger gradient in electric potential near the electrode surface. ${ }^{21}$ This, in turn, led to a larger capacitive current density according to eq 22 . Overall, these results demonstrate that the unequal ion size needs to be accounted for in order to accurately predict the current density for asymmetric electrolytes.

4.1.2. Effect of Asymmetric Valency. Figure 3 shows the CV curves predicted for electrolytes with different valencies, namely

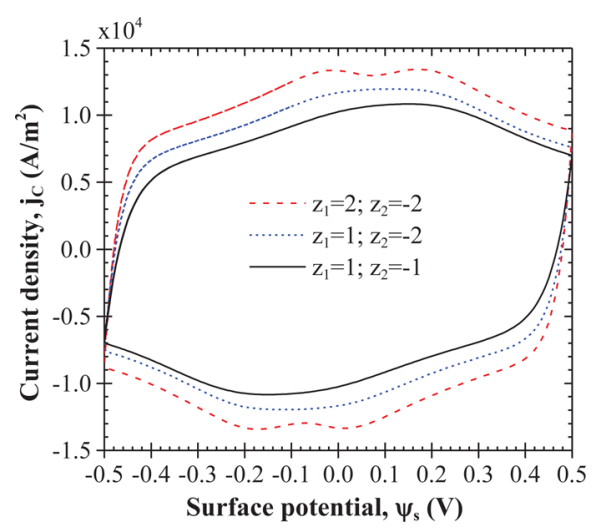

Figure 3. Predicted $j_{\mathrm{C}}$ versus $\psi_{\mathrm{s}}$ curves from CV simulations for three cases with different valencies, namely, (i) $z_{1}=-z_{2}=2$, (ii) $z_{1}=1$ and $z_{2}=-2$, and (iii) $z_{1}=-z_{2}=1$. The model and other parameters were identical to those used to generate the results shown in Figure 2.

(i) $z_{1}=-z_{2}=1$, (ii) $z_{1}=1$ and $z_{2}=-2$, and (iii) $z_{1}=-z_{2}=2$. Here, the ion diameters and diffusion coefficients were identical such that $a_{1}=a_{2}=0.60 \mathrm{~nm}, D_{1}=D_{2}=1.957 \times 10^{-9} \mathrm{~m}^{2} / \mathrm{s}$, and $L_{\mathrm{s}}=0 \mathrm{~m}$. It is evident that the predicted current density $j_{\mathrm{C}}$ for asymmetric valency $z_{1}=1$ and $z_{2}=-2$ lay between those obtained for symmetric valency with $z_{1}=-z_{2}=1$ and $z_{1}=$ $-z_{2}=2$. The relative difference in current density $j_{\mathrm{C}}$ between asymmetric and symmetric electrolytes was about 9\%. Moreover, $j_{C}$ increased with increasing valency $\left|z_{i}\right|$. This can be attributed to the fact that increasing the valency $\left|z_{i}\right|$ led to increasing the amount of charges accumulated near the electrode surface. This, in turn, led to a larger local electric field near the electrode surface and thus larger current density.

4.1.3. Effect of Asymmetric Diffusion Coefficient. Figures 4 show $j_{\mathrm{C}}$ versus $\psi_{\mathrm{s}}$ predicted at (a) low scan rate $v=10^{2} \mathrm{~V} / \mathrm{s}$ and (b) high scan rate $v=10^{5} \mathrm{~V} / \mathrm{s}$ for three cases with different ion diffusion coefficients, namely (i) $D_{1}=D_{2}=2.69 D_{0}$, (ii) $D_{1}=D_{0}$ and $D_{2}=2.69 D_{0}$, and (iii) $D_{1}=D_{2}=D_{0}$ with $D_{0}=1.957 \times$ $10^{-9} \mathrm{~m}^{2} / \mathrm{s}$. The ion diameter and valency were identical, namely, $a_{1}=a_{2}=0.60 \mathrm{~nm}$ and $z_{1}=-z_{2}=1$, while the electrode thickness was zero, i.e., $L_{s}=0 \mathrm{~m}$. Figure 4 a demonstrates that, at low scan rate $v=10^{2} \mathrm{~V} / \mathrm{s}$, the predicted $\mathrm{CV}$ curves overlapped for all three cases despite differences in diffusion coefficients. Moreover, the CV curves were symmetric about the $j_{C}=0$ axis. Note that similar phenomena were also observed in $\mathrm{CV}$ simulations for binary and symmetric electrolytes. ${ }^{9}$ This can be attributed to the fact that ion transport is diffusion-independent at low scan rates ${ }^{9}$ and is controlled by the electric field. On the other hand, Figure $4 \mathrm{~b}$ (a)

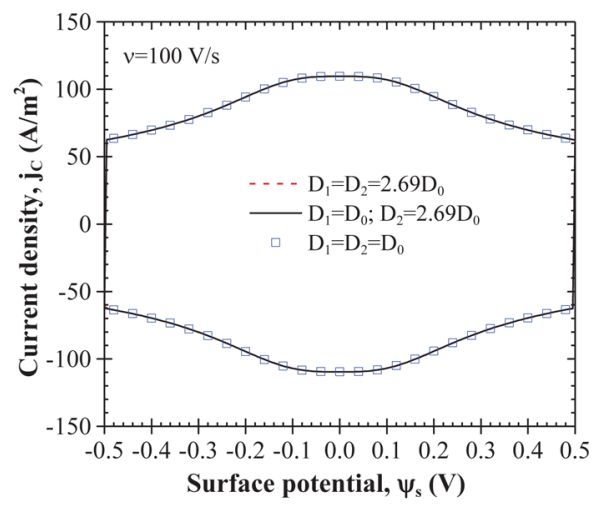

(b)

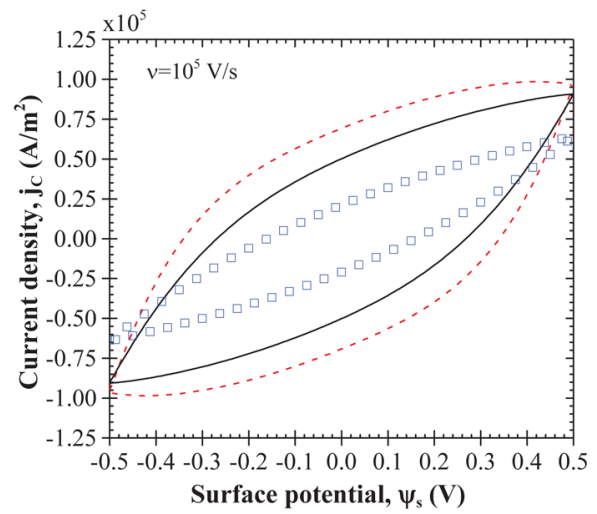

Figure 4. Predicted $j_{\mathrm{C}}$ versus $\psi_{\mathrm{s}}$ curves from CV simulations for (a) $v=102 \mathrm{~V} / \mathrm{s}$ and $(\mathrm{b}) v=10^{5} \mathrm{~V} / \mathrm{s}$. Three cases with different ion diffusion coefficients were considered, namely, (i) $D_{1}=D_{2}=2.69 D_{0}$, (ii) $D_{1}=D_{0}$ and $D_{2}=2.69 D_{0}$, and (iii) $D_{1}=D_{2}=D_{0}$ along with $D_{0}=$ $1.957 \times 10^{-9} \mathrm{~m}^{2} / \mathrm{s}$. The model and other parameters were identical to those used to generate the results shown in Figure 2.

demonstrates that the predicted CV curves became distorted at large scan rate $v=10^{5} \mathrm{~V} / \mathrm{s}$. Here, the predicted current density $j_{C}$ and thus the capacitance increased with increasing ion diffusion coefficient $D_{1}$ or $D_{2}$. Indeed, larger diffusion coefficients enable ions to better follow the rapid variations in electric potential. ${ }^{9}$ These results demonstrate that asymmetric ion diffusion coefficients must be accounted for in $\mathrm{CV}$ simulations at large scan rates but have no effect on $\mathrm{CV}$ curves at low scan rates.

4.2. Dimensional Analysis. Table 1 summarizes the different values of $v, L, T, \psi_{\max }, \psi_{\min }, a_{i}, D_{i}, z_{i}$ and $c_{i \infty}$ for four different cases of binary asymmetric electrolytes considered. Parameters used in Case 1 corresponded to aqueous asymmetric electrolyte $\mathrm{KOH} .{ }^{9,24,74}$ Note that the dimensionless numbers for all four cases were identical, namely, $v^{*}=1.8 \times 10^{-5}, \psi_{\max }^{*}=$ $-\psi_{\text {min }}^{*}=19.5, L^{*}=3.29 \times 10^{5}, a_{1}^{*}=2.17, v_{\mathrm{p} 1}=0.346, v_{\mathrm{p} 2}=0.26$, $D_{2}^{*}=2.5, z_{2}^{*}=-1, \sigma_{\mathrm{s}}^{*} \rightarrow \infty$, and $L_{\mathrm{s}}^{*}=0$. Figures 5a shows the predicted $j_{\mathrm{C}}$ versus $\psi_{\mathrm{s}}$ curves obtained for these four cases. Results were obtained by numerically solving the generalized MPNP model with a Stern layer (eqs 7-12). Figure 5a indicates that the $\mathrm{CV}$ curves were significantly different in these four cases. However, Figure $5 \mathrm{~b}$ shows that the same data plotted in terms of dimensionless current density $j_{C}^{*}$ versus dimensionless surface potential $\psi_{\mathrm{s}}^{*}$ collapsed on a single CV curve. Note that such self-similar behaviors were also observed for cases with $z_{2}^{*}=-2$ and $z_{2}^{*}=-3$. Overall, these results illustrated that the governing equations and the boundary and initial conditions were properly scaled by parameters defined in 
Table 1. Parameters Used in CV Simulations Reported in Figure $5^{a}$

\begin{tabular}{|c|c|c|c|c|c|c|c|c|c|c|c|c|}
\hline & $v(\mathrm{~V} / \mathrm{s})$ & $L(\mu \mathrm{m})$ & $T(\mathrm{~K})$ & $\psi_{\max }=-\psi_{\min }(\mathrm{V})$ & $\varepsilon_{\mathrm{r}}$ & $a_{1}(\mathrm{~nm})$ & $a_{2}(\mathrm{~nm})$ & $D_{1}\left(\mathrm{~m}^{2} / \mathrm{s}\right)$ & $D_{2}\left(\mathrm{~m}^{2} / \mathrm{s}\right)$ & $z_{1}$ & $z_{2}$ & $c_{1 \infty}(\mathrm{mol} / \mathrm{L})$ \\
\hline Case 1 & $10^{4}$ & 100 & 298 & 0.5 & 78.5 & 0.66 & 0.60 & $2 \times 10^{-9}$ & $5 \times 10^{-9}$ & 1 & -1 & 1 \\
\hline Case 2 & $2 \times 10^{4}$ & 50 & 298 & 0.5 & 157 & 0.33 & 0.30 & $1 \times 10^{-9}$ & $2.5 \times 10^{-9}$ & 1 & -1 & 8 \\
\hline Case 3 & $1.33 \times 10^{4}$ & 75 & 298 & 0.5 & 104.7 & 0.495 & 0.45 & $1.5 \times 10^{-9}$ & $3.75 \times 10^{-9}$ & 1 & -1 & 2.37 \\
\hline Case 4 & $1.33 \times 10^{4}$ & 75 & 89.4 & 0.15 & 348.9 & 0.495 & 0.45 & $5 \times 10^{-8}$ & $1.25 \times 10^{-9}$ & 1 & -1 & 2.37 \\
\hline
\end{tabular}

${ }^{a}$ In these four cases, the dimensionless numbers were identical and equal to $v^{*}=1.8 \times 10^{-5}, \psi_{\max }^{*}=-\psi_{\min }^{*}=19.5, L^{*}=3.29 \times 10^{5}, a_{1}^{*}=2.17, \nu_{\mathrm{p} 1}=$ $0.346, \nu_{\mathrm{p} 2}=0.26, D_{2}^{*}=2.5, z_{2}^{*}=-1, \sigma_{\mathrm{s}}^{*} \rightarrow \infty$, and $L_{\mathrm{s}}^{*}=0$.

(a)

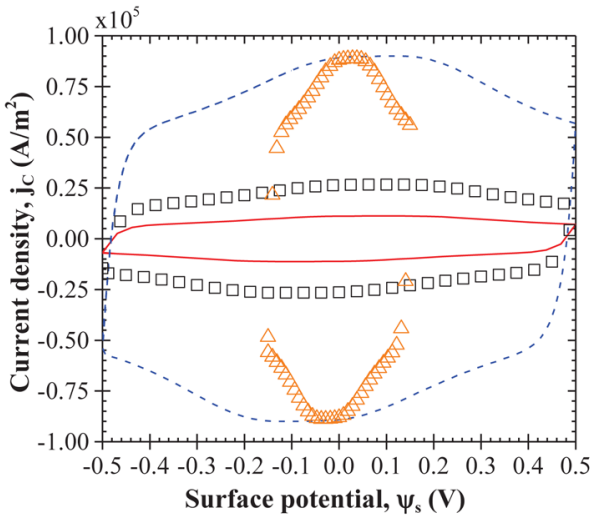

(b)

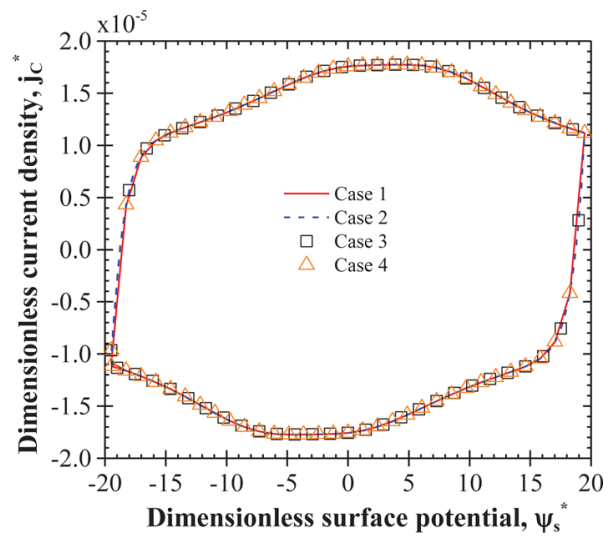

Figure 5. Predicted (a) $j_{\mathrm{C}}$ versus $\psi_{\mathrm{s}}$ curves and (b) $j_{\mathrm{C}}^{*}$ versus $\psi_{\mathrm{s}}^{*}$ curves from CV simulations for four cases with parameters given in Table 1. Results were obtained by numerically solving the generalized MPNP model with a Stern layer (eqs 7-12) for $v^{*}=1.8 \times 10^{-5}, \psi_{\max }^{*}=$ $-\psi_{\text {min }}^{*}=19.5, L^{*}=3.29 \times 10^{5}, a_{1}^{*}=2.17, v_{\mathrm{p} 1}=0.346, v_{\mathrm{p} 2}=0.26$, $D_{2}^{*}=2.5, z_{2}^{*}=-1, \sigma_{\mathrm{s}}^{*} \rightarrow \infty$, and $L_{\mathrm{s}}^{*}=0$.

eqs 13. More importantly, they show that the electric double layer dynamics for binary asymmetric electrolytes near planar electrodes in $\mathrm{CV}$ measurements were governed by 11 dimensionless similarity parameters, namely, $v^{*}, \psi_{\max }^{*} \psi_{\text {min }}^{*}$ $L^{*}, a_{1}^{*}, v_{\mathrm{p} 1}, v_{\mathrm{p} 2}, D_{2}^{*}, z_{2}^{*}, \sigma_{\mathrm{s}}^{*}$, and $L_{\mathrm{s}}^{*}$ given by eq 21 .

Moreover, a dimensionless areal integral capacitance can be defined as

$$
C_{\mathrm{s}}^{*}=\frac{C_{\mathrm{s}}}{z_{1} e N_{\mathrm{A}} D_{1} c_{1, \infty} / \lambda_{D} v}=\frac{1}{\psi_{\text {max }}^{*}-\psi_{\text {min }}^{*}} \oint \frac{j_{\mathrm{C}}^{*}}{2 v^{*}} \mathrm{~d} \psi_{\mathrm{s}}^{*}
$$

where $C_{s}$ is defined in eq 1 . Graphically, $C_{s}^{*}$ corresponds to the area enclosed by the $j_{\mathrm{C}}^{*}$ versus $\psi_{\mathrm{s}}^{*}$ curve. Figure $5 \mathrm{~b}$ demonstrates that $C_{s}^{*}$ depends only on the eleven dimensionless numbers so that $C_{s}^{*}=f\left(v^{*}, \psi_{\max }^{*}, \psi_{\min }^{*}, L^{*}, a_{1}^{*}, v_{\mathrm{p} 1}, v_{\mathrm{p} 2}, D_{2}^{*}, z_{2}^{*}\right.$, $\left.\sigma_{s}^{*}, L_{s}^{*}\right)$. This relation can be used to formulate design rules for

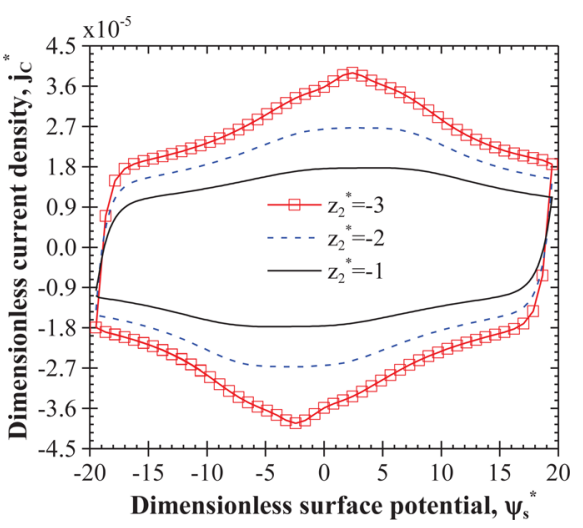

Figure 6. Predicted $j_{\mathrm{C}}^{*}$ versus $\psi_{\mathrm{s}}^{*}$ curves from $\mathrm{CV}$ simulations for three different values of $z_{2}^{*}$, namely, $z_{2}^{*}=-1,-2$, and -3 . The model and other dimensionless numbers were identical to those used to generate the results shown in Figure 5.
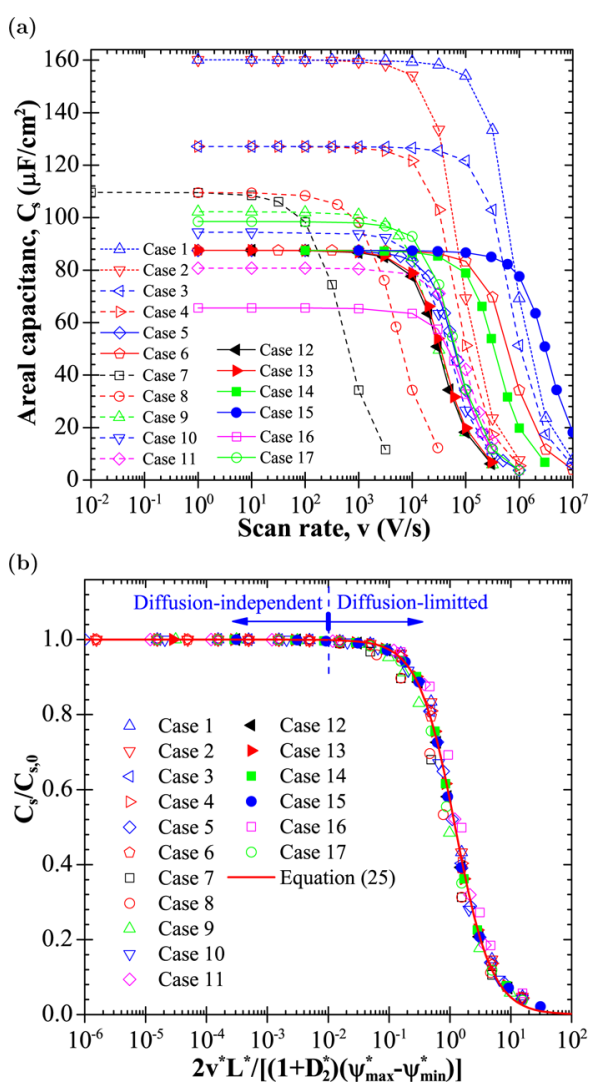

Figure 7. Predicted (a) capacitance $C_{\mathrm{s}}$ as a function of scan rate $v$ and (b) ratio $C_{\mathrm{s}} / C_{\mathrm{s}, 0}$ as a function of $2 v^{*} L^{*} /\left[\left(1+D_{2}^{*}\right)\left(\psi_{\max }^{*}-\psi_{\min }^{*}\right)\right]$ obtained from CV simulations for cases 1 to 17 without accounting for the potential drop across the electrodes (i.e., $\sigma_{\mathrm{s}}^{*} \rightarrow \infty$ or $L_{\mathrm{s}}^{*}=0$ ) with dimensionless parameters $\mathrm{L}^{*}, z_{2}^{*}, \psi_{\max }^{*}=-\psi_{\min }^{*}, D_{2}^{*}, a_{1}^{*}, v_{\mathrm{p} 1}$, and $v_{\mathrm{p} 2}$ summarized in Table 2. 
Table 2. Dimensionless Parameters for 29 Cases of CV Simulations Reported in Figures 7 and 8 along with the Respective Values of Maximum Capacitance $C_{\mathrm{s}, 0}$

\begin{tabular}{|c|c|c|c|c|c|c|c|c|c|c|}
\hline & $L^{*}$ & $z_{2}^{*}$ & $\psi_{\max }^{*}=-\psi_{\min }^{*}$ & $D_{2}^{*}$ & $a_{1}^{*}$ & $\nu_{\mathrm{p} 1}$ & $\nu_{\mathrm{p} 2}$ & $\sigma_{\mathrm{s}}^{*}$ & $L_{\mathrm{s}}^{*}$ & $C_{s, 0}\left(\mu \mathrm{F} / \mathrm{cm}^{2}\right)$ \\
\hline Case 1 & 32900 & -3 & 19.5 & 1 & 2.17 & 0.346 & 0.346 & $\infty$ & 0 & 160.1 \\
\hline Case 2 & 329000 & -3 & 19.5 & 1 & 2.17 & 0.346 & 0.346 & $\infty$ & 0 & 160.1 \\
\hline Case 3 & 32900 & -2 & 19.5 & 1 & 2.17 & 0.346 & 0.346 & $\infty$ & 0 & 127.1 \\
\hline Case 4 & 329000 & -2 & 19.5 & 1 & 2.17 & 0.346 & 0.346 & $\infty$ & 0 & 127.1 \\
\hline Case 5 & 32900 & -1 & 19.5 & 1 & 2.17 & 0.346 & 0.346 & $\infty$ & 0 & 87.5 \\
\hline Case 6 & 329000 & -1 & 19.5 & 1 & 2.17 & 0.346 & 0.346 & $\infty$ & 0 & 87.5 \\
\hline Case 7 & 329000 & -1 & 0.195 & 1 & 2.17 & 0.346 & 0.346 & $\infty$ & 0 & 109.6 \\
\hline Case 8 & 329000 & -1 & 1.95 & 1 & 2.17 & 0.346 & 0.346 & $\infty$ & 0 & 109.5 \\
\hline Case 9 & 329000 & -1 & 9.75 & 1 & 2.17 & 0.346 & 0.346 & $\infty$ & 0 & 102.2 \\
\hline Case 10 & 329000 & -1 & 14.625 & 1 & 2.17 & 0.346 & 0.346 & $\infty$ & 0 & 94.44 \\
\hline Case 11 & 329000 & -1 & 25.35 & 1 & 2.17 & 0.346 & 0.346 & $\infty$ & 0 & 87.77 \\
\hline Case 12 & 329000 & -1 & 19.5 & 0.01 & 2.17 & 0.346 & 0.346 & $\infty$ & 0 & 87.5 \\
\hline Case 13 & 329000 & -1 & 19.5 & 0.1 & 2.17 & 0.346 & 0.346 & $\infty$ & 0 & 87.5 \\
\hline Case 14 & 329000 & -1 & 19.5 & 10 & 2.17 & 0.346 & 0.346 & $\infty$ & 0 & 87.5 \\
\hline Case 15 & 329000 & -1 & 19.5 & 100 & 2.17 & 0.346 & 0.346 & $\infty$ & 0 & 87.5 \\
\hline Case 16 & 329000 & -1 & 19.5 & 1 & 3.06 & 0.97 & 0.26 & $\infty$ & 0 & 65.6 \\
\hline Case 17 & 329000 & -1 & 19.5 & 1 & 1.97 & 0.26 & 0.26 & $\infty$ & 0 & 98.5 \\
\hline Case 18 & 263.1 & -1 & 19.5 & 1 & 2.17 & 0.346 & 0.346 & $1.61 \times 10^{-7}$ & 329 & 87.5 \\
\hline Case 19 & 263.1 & -1 & 19.5 & 1 & 2.17 & 0.346 & 0.346 & $1.61 \times 10^{-4}$ & 329 & 87.5 \\
\hline Case 20 & 263.1 & -1 & 19.5 & 1 & 2.17 & 0.346 & 0.346 & $1.61 \times 10^{-1}$ & 329 & 87.5 \\
\hline Case 21 & 263.1 & -1 & 19.5 & 1 & 2.17 & 0.346 & 0.346 & $\infty$ & 329 & 87.5 \\
\hline Case 22 & 32900 & -1 & 19.5 & 1 & 2.17 & 0.346 & 0.346 & $1.61 \times 10^{-4}$ & 0 & 87.5 \\
\hline Case 23 & 32900 & -1 & 19.5 & 1 & 2.17 & 0.346 & 0.346 & $1.61 \times 10^{-4}$ & 329 & 87.5 \\
\hline Case 24 & 32900 & -1 & 19.5 & 1 & 2.17 & 0.346 & 0.346 & $1.61 \times 10^{-4}$ & 3290 & 87.5 \\
\hline Case 25 & 32900 & -1 & 19.5 & 1 & 2.17 & 0.346 & 0.346 & $1.61 \times 10^{-4}$ & 98400 & 87.5 \\
\hline Case 26 & 16400 & -3 & 19.5 & 100 & 2.17 & 0.346 & 0.346 & $1.61 \times 10^{-4}$ & 233000 & 101.4 \\
\hline Case 27 & 32900 & -2 & 19.5 & 10 & 2.17 & 0.346 & 0.346 & $1.61 \times 10^{-4}$ & 98400 & 97.0 \\
\hline Case 28 & 403000 & -2 & 19.5 & 1 & 2.17 & 0.346 & 0.346 & 0.0566 & 121000 & 97.0 \\
\hline Case 29 & 403000 & -2 & 19.5 & 10 & 2.17 & 0.346 & 0.346 & 0.0566 & 121000 & 97.0 \\
\hline
\end{tabular}

EDLCs with asymmetric electrolytes. However, finding this multidimensional function falls beyond the scope of this study.

Finally, Figure 6 shows the predicted $j_{\mathrm{C}}^{*}$ versus $\psi_{\mathrm{s}}^{*}$ curves for three cases with $z_{2}^{*}=-1,-2$, and -3 , respectively. The model and the other ten dimensionless numbers were identical to those used to generate the results shown in Figure 5. It is evident that $j_{\mathrm{C}}^{*}$ and therefore $C_{\mathrm{s}}^{*}$ increased as $z_{2}^{*}$ decreased (i.e., increasing $\left.\left|z_{2}^{*}\right|\right)$ due to the increase in the amount of charges accumulated at the electrode surface which induced a larger electric current.

4.3. Capacitance versus Scan Rate. Figure 7a shows the double layer areal integral capacitance $C_{s}$ predicted from $\mathrm{CV}$ simulations and estimated using eq 1 as a function of scan rate $v$ ranging from $10^{-2}$ to $10^{7} \mathrm{~V} / \mathrm{s}$ for 17 cases of binary asymmetric electrolytes without accounting for the potential drop across the electrodes. This corresponds to cases with infinitely large electrode electrical conductivity $\left(\sigma_{s} \rightarrow \infty \mathrm{S} / \mathrm{m}\right)$ or zero electrode thickness $\left(L_{s} \rightarrow 0 \mathrm{~m}\right)$. Table 2 summarizes the values of the 10 dimensionless parameters $L^{*}, z_{2}^{*}, \psi_{\max }^{*}=-\psi_{\min }^{*} D_{2}^{*}$, $a_{1}^{*}, v_{\mathrm{p} 1}, v_{\mathrm{p} 2}, \sigma_{\mathrm{s}}^{*}$, and $L_{\mathrm{s}}^{*}$ along with the maximum capacitances $C_{\mathrm{s}, 0}$ corresponding to the plateau observed in $C_{\mathrm{s}}$ versus $v$ at low scan rates. Note that $C_{s, 0}$ depends on a variety of parameters including $\psi_{\mathrm{s}}, a_{1}, a_{2}, z_{1}, z_{2}$, and $c_{1 \infty}$. However, it falls beyond the scope of this manuscript to find an analytical expression relating these parameters for asymmetric electrolytes. A remarkably broad range of values were considered for each dimensionless number. It is evident that these $C_{\mathrm{s}}$ versus $v$ curves as well as $C_{\mathrm{s}, 0}$ were significantly different from one another. Figure $7 \mathrm{~b}$ shows the same data as those shown in Figure 7a but plotted in terms of $C_{\mathrm{s}} / C_{\mathrm{s}, 0}$ as a function of $2 v^{*} L^{*} /\left[\left(1+D_{2}^{*}\right)\right]\left(\psi_{\max }^{*}-\psi_{\min }^{*}\right)$. It is interesting to note that all the curves collapsed on a single line, irrespective of the different values of $L^{*}, z_{2}^{*}, \psi_{\max }^{*}=-\psi_{\min }^{*}, D_{2}^{*}$, $a_{1}^{*}, v_{\mathrm{p} 1}$, and $v_{\mathrm{p} 2}$. The Supporting Information presents the effects of each parameter separately. To the best of our knowledge, the present study is the first to identify this selfsimilar behavior of electric double layer integral capacitance in $\mathrm{CV}$ measurements with binary asymmetric electrolytes. Fitting the dimensionless data shown in Figure $7 \mathrm{~b}$ yields the following correlation with a coefficient of determination equal to 0.999

$$
\frac{C_{\mathrm{s}}}{C_{\mathrm{s}, 0}}=\frac{1}{1+\left[\frac{2 v^{*} L^{*} /\left(1+D_{2}^{*}\right)\left(\psi_{\max }^{*}-\psi_{\min }^{*}\right)}{1.22}\right]^{1.44}}
$$

The dimensionless abscissa in Figure $7 \mathrm{~b}$ can be interpreted as the ratio of two time scales,

$$
\frac{2 v^{*} L^{*}}{\left(1+D_{2}{ }^{*}\right)\left(\psi_{\max }^{*}-\psi_{\min }^{*}\right)}=\frac{2 \lambda_{\mathrm{D}} L /\left(D_{1}+D_{2}\right)}{\left(\psi_{\max }-\psi_{\min }\right) / v}=\frac{\tau_{\mathrm{RC}}}{\tau_{\mathrm{CV}}}
$$

where $\tau_{\mathrm{RC}}$ is the "RC time scale" for binary asymmetric electrolytes corresponding to the characteristic time of ions' electrodiffusion $^{77}$ and $\tau_{\mathrm{CV}}$ is the half cycle period of $\mathrm{CV}$ measurements. They are expressed as

$$
\tau_{\mathrm{RC}}=\sqrt{\tau_{\mathrm{L}} \tau_{\mathrm{D}}}=\frac{\lambda_{\mathrm{D}} L}{\left(D_{1}+D_{2}\right) / 2} \quad \text { and } \quad \tau_{\mathrm{CV}}=\frac{\psi_{\text {max }}-\psi_{\text {min }}}{v}
$$


Here, $\tau_{L}=2 L^{2} /\left(D_{1}+D_{2}\right)$ is the time scale for ion diffusion in binary asymmetric electrolytes and $\tau_{D}=2 \lambda_{D}^{2} /\left(D_{1}+D_{2}\right)$ has been termed as the "charge relaxation time scale". ${ }^{77}$ Note that $\tau_{\mathrm{RC}}$ reduces to the corresponding "RC time scale" of binary symmetric electrolytes when $D_{2}^{*}=1$ as considered in refs 22 , 23 , and 77 . Figure $7 \mathrm{~b}$ indicates that two regimes can be clearly identified: (i) $2 v^{*} L^{*} /\left[\left(D_{1}+D_{2}^{*}\right)\left(\psi_{\max }^{*}-\psi_{\text {min }}^{*}\right)\right] \ll 1$ or $\tau_{\mathrm{RC}} \ll \tau_{\mathrm{CV}}$ corresponds to the quasi-equilibrium or ion diffusion-independent regime and (ii) $2 v^{*} L^{*} /\left[\left(D_{1}+D_{2}^{*}\right)\right.$ $\left.\left(\psi_{\max }^{*}-\psi_{\min }^{*}\right)\right] \ll 1$ or $\tau_{\mathrm{RC}} \ll \tau_{\mathrm{CV}}$, corresponds to ion diffusionlimited regime.

4.4. Effect of the Electrode. The above simulations ignored the potential drop across the electrodes. Figure 8a
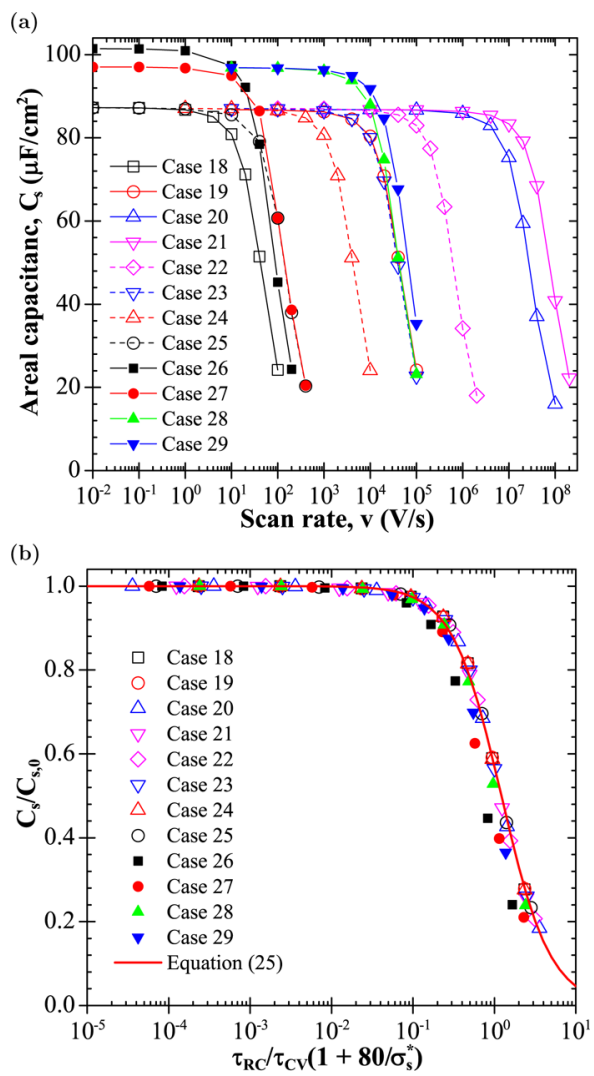

Figure 8. Predicted (a) capacitance $C_{\mathrm{s}}$ as a function of scan rate $v$ and (b) ratio $C_{\mathrm{s}} / C_{\mathrm{s}, 0}$ as a function of $T_{\mathrm{RC}} / T_{\mathrm{CV}}\left(1+80 / \sigma_{\mathrm{s}}^{*}\right)$ obtained from CV simulations for cases 18 to 29 with dimensionless parameters $L^{*}$, $z_{2}^{*}, \psi_{\max }^{*}=-\psi_{\min }^{*}, D_{2}^{*}, a_{1}^{*}, v_{\mathrm{p} 1}, v_{\mathrm{p} 2}, \sigma_{\mathrm{s}}^{*}$, and $L_{\mathrm{s}}^{*}$ summarized in Table 2 .

shows the double-layer areal integral capacitance $C_{\mathrm{s}}$ predicted from $\mathrm{CV}$ simulations as a function of scan rate $v$ ranging from $10^{-2}$ to $10^{8} \mathrm{~V} / \mathrm{s}$ for 12 cases accounting for charge transport in the electrodes. Table 2 summarizes the values of the eleven dimensionless parameters $L^{*}, z_{2}^{*}, \psi_{\max }^{*}=-\psi_{\min }^{*}, D_{2}^{*}, a_{1}^{*}, v_{\mathrm{p} 1}, v_{\mathrm{p} 2}$, $\sigma_{\mathrm{s}}^{*}$, and $L_{\mathrm{s}}^{*}$ along with the maximum capacitances $C_{s, 0}$ for cases 18 to 29. It is evident that the curves $C_{s}$ versus $v$ were significantly different from one another due to the broad range of parameters considered. Figure $8 \mathrm{~b}$ shows the same data as those shown in Figure 8a but plotted in terms of $C_{\mathrm{s}} / C_{\mathrm{s}, 0}$ as a function of $\tau_{\mathrm{RC}} / \tau_{\mathrm{CV}}\left(1+80 / \sigma_{\mathrm{s}}^{*}\right)$. The fitted curve given by eq 25 for cases ignoring the potential drop across the electrodes $\left(\sigma_{s}^{*} \rightarrow \infty\right.$ or $\left.L_{s}^{*} \rightarrow 0\right)$ was also shown in Figure $8 \mathrm{~b}$ for comparison purposes. It is interesting to note that all the curves collapsed on a single line, irrespective of the different values of $L^{*}, z_{2}^{*}, \psi_{\max }^{*}=-\psi_{\min }^{*}, D_{2}^{*}, a_{1}^{*}, v_{\mathrm{p} 1}, v_{\mathrm{p} 2}, \sigma_{\mathrm{s}}^{*}$, and $L_{\mathrm{s}}^{*}$.

The dimensionless abscissa in Figure $7 \mathrm{~b}$ can be interpreted as

$$
\frac{\tau_{\mathrm{RC}}}{\tau_{\mathrm{CV}}}\left(1+\frac{80}{\sigma_{\mathrm{s}}^{*}}\right)=\frac{\tau_{\mathrm{RC}}}{\tau_{\mathrm{CV}}}\left(1+80 \frac{\tau_{\mathrm{s}}}{\tau_{\mathrm{d}}}\right)
$$

where $\sigma_{\mathrm{s}}^{*}=\tau_{\mathrm{d}} / \tau_{\mathrm{s}}$ represents the ratio of characteristic charge transport time scale in the electrolyte $\tau_{\mathrm{d}}$ to that in the electrode $\tau_{s}$ as discussed previously. Note that when $\sigma_{\mathrm{s}}^{*}$ is very large (e.g., $\left.\sigma_{s}^{*} \gg 80\right)$, the charge transport in the electrode is much faster than that in the electrolyte. Then, it suffices to simulate the electrolyte in CV simulations.

\section{CONCLUSIONS}

This paper developed a MPNP model for simulating electric double layer dynamics. This model was derived from first principles based on excess chemical potential and Langmuir activity coefficient accounting simultaneously for (1) asymmetric electrolytes with (2) multiple ion species of (3) finite ion size, and (4) Stern and diffuse layers. This study established that asymmetric ion diameters and valencies must be accounted for in CV simulations with asymmetric electrolytes for all scan rates. By contrast, asymmetric ion diffusion coefficient affected the CV curves only at large scan rates.

Dimensional analysis of the governing equations was also performed for $\mathrm{CV}$ measurements with planar electrodes. Eleven dimensionless numbers given by eq 21 were identified to govern the $\mathrm{CV}$ measurements of electric double layer in binary asymmetric electrolytes between two identical planar electrodes of finite thickness. For the first time, a self-similar behavior was identified for the electric double layer integral capacitance estimated from CV measurements with binary asymmetric electrolytes and planar electrodes. The physical interpretations were also provided. This model provides a theoretical framework to investigate more complex situations with asymmetric electrolytes and/or multiple ion species such as redox reactions in pseudocapacitors and the interplay of Faradaic and capacitive currents encountered in fast-scan cyclic voltammetry.

\section{ASSOCIATED CONTENT}

\section{S Supporting Information}

Results of full domain and half domain simulations as well as the scaling analysis for interelectrode distance, potential window, asymmetric diffusion coefficients, and ion diameter. This material is available free of charge via the Internet at http://pubs.acs.org.

\section{AUTHOR INFORMATION}

\section{Corresponding Author}

*Phone: +1 (310)-206-5598; Fax: +1 (310)-206-2302; E-mail: pilon@seas.ucla.edu.

\section{Notes}

The authors declare no competing financial interest.

\section{ACKNOWLEDGMENTS}

This material is based upon work supported as part of the Molecularly Engineered Energy Materials, an Energy Frontier Research Center funded by the U.S. Department of Energy, Office of Science, Office of Basic Energy Sciences under Award Number DE-SC0001342. 


\section{REFERENCES}

(1) Bard, A. J.; Faulkner, L. R. Electrochemical Methods: Fundamentals and Applications; John Wiley \& Sons: New York, 2001.

(2) Compton, R. G.; Banks, C. E. Understanding Voltammetry; World Scientific: Hackensack, NJ, 2007.

(3) Greff, R.; Peat, R.; Peter, L. M.; Pletcher, D.; Robinson, J. Instrumental Methods in Electrochemistry; Southampton Electrochemistry Group: Chichester, UK, 1985.

(4) Wang, J.; Polleux, J.; Lim, J.; Dunn, B. Pseudocapacitive Contributions to Electrochemical Energy Storage in $\mathrm{TiO}_{2}$ (Anatase) Nanoparticles. J. Phys. Chem. C 2007, 111, 14925-14931.

(5) Brezesinski, T.; Wang, J.; Polleux, J.; Dunn, B.; Tolbert, S. H. Templated Nanocrystal-Based Porous $\mathrm{TiO}_{2}$ Films for Next-Generation Electrochemical Capacitors. J. Am. Chem. Soc. 2009, 131, 1802-1809.

(6) Brezesinski, T.; Wang, J.; Tolbert, S. H.; Dunn, B. Ordered Mesoporous $\alpha-\mathrm{MoO}_{3}$ with Iso-oriented Nanocrystalline Walls for Thin-Film Pseudocapacitors. Nat. Mater. 2010, 9, 146-151.

(7) Pech, D.; Brunet, M.; Durou, H.; Huang, P.; Mochalin, V.; Gogotsi, Y.; Taberna, P. L.; Simon, P. Ultrahigh-Power MicrometreSized Supercapacitors Based on Onion-like Carbon. Nat. Nanotechnol. 2010, 5, 651-654.

(8) Lee, S. W.; Yabuuchi, N.; Gallant, B. M. High-Power Lithium Batteries from Functionalized Carbon-Nanotube Electrodes. Nat. Nanotechnol. 2010, 5, 531-537.

(9) Wang, H.; Pilon, L. Physical Interpretation of Cyclic Voltammetry for Measuring Electric Double Layer Capacitances. Electrochim. Acta 2012, 64, 130-139.

(10) Kornyshev, A. A. Double-Layer in Ionic Liquids: Paradigm Change? J. Phys. Chem. B 2007, 111, 5545-5557.

(11) Kilic, M. S.; Bazant, M. Z.; Ajdari, A. Steric Effects in the Dynamics of Electrolytes at Large Applied Voltages. I. Double-Layer Charging. Phys. Rev. E 2007, 75, 021502.

(12) Bazant, M. Z.; Storey, B. D.; Kornyshev, A. A. Double Layer in Ionic Liquids: Overscreening Versus Crowding. Phys. Rev. Lett. 2011, 106, 046102.

(13) Wang, H.; Varghese, J.; Pilon, L. Simulation of Electric Double Layer Capacitors with Mesoporous Electrodes: Effects of Morphology and Electrolyte Permittivity. Electrochim. Acta 2011, 56, 6189-6197.

(14) Wang, H.; Pilon, L. Accurate Simulation of Electric Double Layer Capacitance for Ultramicroelectrodes. J. Phys. Chem. C 2011, $115,16711-16719$.

(15) Varghese, J.; Wang, H.; Pilon, L. Simulating Electric Double Layer Capacitance of Mesoporous Electrodes with Cylindrical Pores. J. Electrochem. Soc. 2011, 158, 1106-1114.

(16) Wang, H.; Pilon, L. Mesoscale Modeling of Electric Double Layer Capacitors with Three-Dimensional Ordered Structures. J. Power Sources 2013, 221, 252-260.

(17) Wang, H.; Pilon, L. Intrinsic Limitations of Impedance Measurements in Determining Electric Double Layer Capacitances. Electrochim. Acta 2012, 63, 55-63.

(18) Baker-Jarvis, J.; Riddle, B.; Young, A. M. Ion Dynamics Near Charged Electrodes with Excluded Volume Effect. IEEE T. Dielect. El. In. 1999, 6, 226-235.

(19) Kilic, M. S.; Bazant, M. Z.; Ajdari, A. Steric Effects in the Dynamics of Electrolytes at Large Applied Voltages. II. Modified Poisson-Nernst-Planck equations. Phys. Rev. E 2007, 75, 021503.

(20) Chu, K. T.; Bazant, M. Z. Surface Conservation Laws at Microscopically Diffuse Interfaces. J. Colloid Interface Sci. 2007, 315, 319-329.

(21) Bazant, M. Z.; Kilic, M. S.; Storey, B. D.; Ajdari, A. Towards an Understanding of Induced-Charge Electrokinetics at Large Applied Voltages in Concentrated Solutions. Adv. Colloid Interfac. 2009, 152, $48-88$.

(22) Olesen, L. H.; Bazant, M. Z.; Bruus, H. Strongly Nonlinear Dynamics of Electrolytes in Large ac Voltages. Phys. Rev. E 2010, 82, 011501.

(23) Zhao, H. Diffuse-charge Dynamics of Ionic Liquids in Electrochemical Systems. Phys. Rev. E 2011, 84, 051504.
(24) Lide, D. R., Ed. CRC Handbook of Chemistry and Physics, 90th ed.; CRC Press/Taylor \& Francis: Boca Raton, FL, 2010.

(25) Galinski, M.; Lewandowski, A.; Stepniak, I. Ionic Liquids as Electrolytes. Electrochim. Acta 2006, 51, 5567-5580.

(26) Lin, R.; Taberna, P.-L.; Fantini, S.; Presser, V.; Pérez, C. R.; Malbosc, F.; Rupesinghe, N. L.; Teo, K. B. K.; Gogotsi, Y.; Simon, P. Capacitive Energy Storage from -50 to $100{ }^{\circ} \mathrm{C}$ Using an Ionic Liquid Electrolyte. J. Phys. Chem. Lett. 2011, 2, 2396-2401.

(27) Li, S.; Feng, G.; Fulvio, P. F.; Hillesheim, P. C.; Liao, C.; Dai, S.; Cummings, P. T. Molecular Dynamics Simulation Study of the Capacitive Performance of a Binary Mixture of Ionic Liquids Near an Onion-like Carbon Electrode. J. Phys. Chem. Lett. 2012, 3, 2465-2469.

(28) Palm, R.; Kurig, H.; Tõnurist, K.; Jänes, A.; Lust, E. Is the Mixture of 1-Ethyl-3-methylimidazolium Tetrafluoroborate and 1Butyl-3-methylimidazolium Tetrafluoroborate Applicable as Electrolyte in Electrical Double Layer Capacitors. Electrochem. Commun. 2012, 22, 203-206.

(29) Biesheuvel, P. M.; Fu, Y.; Bazant, M. Z. Electrochemistry and Capacitive Charging of Porous Electrodes in Asymmetric Multicomponent Electrolytes. Russ. J. Electrochem+ 2012, 48, 580-592.

(30) Zhao, R.; van Soestbergen, M.; Rijnaarts, H. H. M.; van der Wal, A.; Bazant, M. Z.; Biesheuvel, P. M. Time-Dependent Ion Selectivity in Capacitive Charging of Porous Electrodes. J. Colloid Interface Sci. 2012, $384,38-44$

(31) Lopez-García, J. J.; Aranda-Rascón, M. J.; Horno, J. Excluded Volume Effect on the Electrophoretic Mobility of Colloidal Particles. J. Colloid Interface Sci. 2008, 323, 146-152.

(32) Aranda-Rascon, M. J.; Grosse, C.; Lopez-Garcia, J. J.; Horno, J. Electrokinetics of Suspended Charged Particles Taking into Account the Excluded Volume Effect. J. Colloid Interface Sci. 2009, 335, 250256.

(33) Lopez-García, J. J.; Horno, J.; Grosse, C. Equilibrium Properties of Charged Spherical Colloidal Particles Suspended in Aqueous Electrolytes: Finite Ion Size and Effective Ion Permittivity Effects. J. Colloid Interface Sci. 2012, 380, 213-221.

(34) Lopez-García, J. J.; Aranda-Rascón, M. J.; Horno, J. Electrical Double Layer Around a Spherical Colloid Particle: The Excluded Volume Effect. J. Colloid Interface Sci. 2007, 316, 196-201.

(35) Lopez-García, J. J.; Horno, J. Poisson-Boltzmann Description of the Electrical Double Layer Including Ion Size Effects. Langmuir 2011, 27, 13970-13974.

(36) Lopez-García, J. J.; Aranda-Rascón, M. J.; Grosse, C.; Horno, J. Equilibrium Electric Double Layer of Charged Spherical Colloidal Particles: Effect of Different Distances of Minimum Ion Approach to the Particle Surface. J. Phys. Chem. B 2010, 114, 7548-7556.

(37) Lopez-García, J. J.; Aranda-Rascón, M. J.; Grosse, C.; Horno, J. Electrokinetics of Charged Spherical Colloidal Particles Taking into Account the Effect of Ion Size Constraints. J. Colloid Interface Sci. 2011, 356, 325-330.

(38) Valleau, J. P.; Torrie, G. M. The Electrical Double Layer. III. Modified Gouy-Chapman Theory with Unequal Ion Sizes. J. Chem. Phys. 1982, 76, 4623-4630.

(39) Yu, J.; Aguilar-Pineda, G. E.; Antillon, A.; Dong, S.-H.; LozadaCassou, M. The Effects of Unequal Ionic Sizes for an Electrolyte in A Charged Slit. J. Colloid Interface Sci. 2006, 295, 124-134.

(40) Alijó, P. H. R.; Tavares, F. W.; Biscaia, E. C., Jr. Double Layer Interaction Between Charged Parallel Plates Using a Modified Poisson-Boltzmann Equation to Include Size Effects and Ion Specificity. Colloids Surfaces A 2012, 412, 29-35.

(41) Wipf, D. O.; Kristensen, E. W.; Deakin, M. R.; Wightman, R. M. Fast-Scan Cyclic Voltammetry as a Method to Measure Rapid Heterogeneous Electron-Transfer Kinetics. Anal. Chem. 1988, 60, 306-310.

(42) Robinson, D. L.; Hermans, A.; Seipel, A. T.; Wightman, R. M. Monitoring Rapid Chemical Communication in the Brain. Chem. Rev. 2008, 108, 2554-2584.

(43) Perry, M.; Li, Q.; Kennedy, R. T. Review of Recent Advances in Analytical Techniques for the Determination of Neurotransmitters. Anal. Chim. Acta 2009, 653, 1-22. 
(44) Davidson, J. D.; Goulbourne, N. C. Nonlinear Capacitance and Electrochemical Response of Ionic Liquid-Ionic Polymers. J. Appl. Phys. 2011, 109, 1-14.

(45) Eisenberg, B.; Hyon, Y.; Liu, C. Energy Variational Analysis of Ions in Water and Channels: Field Theory for Primitive Models of Complex Ionic Fluids. J. Chem. Phys. 2010, 133 (1-23), 104104.

(46) Eisenberg, B. Multiple Scales in the Simulation of Ion Channels and Proteins. J. Phys. Chem. C 2010, 114, 20719-20733.

(47) Eisenberg, B. Crowded Charges in Ion Channels. In Advances in Chemical Physics; Rice, S. A., Dinner, A. R., Eds.; John Wiley \& Sons: Hoboken, NJ, 2012; Vol. 148, pp 77-223.

(48) Horng, T.-L.; Lin, T.-C.; Liu, C.; Eisenberg, B. PNP Equations with Steric Effects: A Model of Ion Flow Through Channels. J. Phys. Chem. B 2012, 116, 11422-11441.

(49) Lu, B.; Zhou, Y. C. Poisson-Nernst-Planck Equations for Simulating Biomolecular Diffusion-reaction Processes II: Size Effects on Ionic Distributions and Diffusion-Reaction Rates. Biophys. J. 2012, 100, 2475-2485.

(50) Zhou, Y. C. Electrodiffusion of Lipids on Membrane Surfaces. J. Chem. Phys. 2012, 136, 205103.

(51) Borukhov, I.; Andelman, D.; Orland, H. Steric Effects in Electrolytes: A Modified Poisson-Boltzmann Equation. Phys. Rev. Lett. 1997, 79, 435-438.

(52) Borukhov, I.; Andelman, D.; Orland, H. Adsorption of Large Ions from an Electrolyte Solution: A Modified Poisson-Boltzmann Equation. Electrochim. Acta 2000, 46, 221-229.

(53) Silalahi, A. R. J.; Boschitsch, A. H.; Harris, R. C.; Fenley, M. O. Comparing the Predictions of the Nonlinear Poisson-Boltzmann Equation and the Ion Size-Modified Poisson-Boltzmann Equation for a Low-dielectric Charged Spherical Cavity in an Aqueous Salt Solution. J. Chem. Theory Comput. 2010, 6, 3631-3639.

(54) Chu, V. B.; Bai, Y.; Lipfert, J.; Herschlag, D.; Doniach, S. Evaluation of Ion Binding to DNA Duplexes Using a Size-modified Poisson-Boltzmann Theory. Biophys. J. 2007, 93, 3202-3209.

(55) Grochowski, P.; Trylska, J. Continuum Molecular Electrostatics, Salt Effects, and Counterion Binding - A Review of the PoissonBoltzmann Theory and Its Modifications. Biopolymers 2008, 89, 93113.

(56) Biesheuvel, P. M.; Lyklema, J. Sedimentation-Diffusion Equilibrium of Binary Mixtures of Charged Colloids Including Volume Effects. J. Phys.: Condens. Matter 2005, 17, 6337-6352.

(57) Biesheuvel, P. M.; Leermakers, F. A. M.; Stuart, M. A. C. SelfConsistent Field Theory of Protein Adsorption in a Non-Gaussian Polyelectrolyte Brush. Phys. Rev. E 2006, 73, 011802.

(58) Biesheuvel, P. M.; van Soestbergen, M. Counterion Volume Effects in Mixed Electrical Double Layers. J. Colloid Interface Sci. 2007, $316,490-499$.

(59) Tresset, G. Generalized Poisson-Fermi Formalism for Investigating Size Correlation Effects with Multiple Ions. Phys. Rev. E 2008, 78, 061506.

(60) Li, B. Continuum Electrostatics for Ionic Solutions with Nonuniform Ionic Sizes. Nonlinearity 2009, 22, 811-833.

(61) Zhou, S.; Wang, Z.; Li, B. Mean-Field Description of Ionic Size Effects with Nonuniform Ionic Sizes: A Numerical Approach. Phys. Rev. E 2011, 84, 021901.

(62) Wen, J.; Zhou, S.; Xu, Z.; Li, B. Competitive Adsorption and Ordered Packing of Counterions Near Highly Charged Surfaces: From Mean-Field Theory to Monte Carlo Simulations. Phys. Rev. E 2012, 85, 041406.

(63) Bikerman, J. J. Structure and Capacity of Electrical Double Layer. Philos. Mag. 1942, 33, 384-397.

(64) Hunter, R. J. Foundations of Colloid Science, 2nd ed.; Oxford University Press: Oxford, UK, 2001.

(65) Aranda-Rascon, M. J.; Grosse, C.; Lopez-Garcia, J. J.; Horno, J. Influence of the Finite Ion Size on the Predictions of the Standard Electrokinetic Model: Frequency Response. J. Colloid Interface Sci. 2009, 336, 857-864.
(66) Cervera, J.; García-Morales, V.; Pellicer, J. Ion Size Effects on the Electrokinetic Flow in Nanoporous Membranes Caused by Concentration Gradients. J. Phys. Chem. B 2003, 107, 8300-8309.

(67) Cervera, J.; Ramírez, P.; Manzanares, J. A.; Mafé, S. Incorporating Ionic Size in the Transport Equations for Charged Nanopores. Microfluid. Nanofluid. 2010, 9, 41-53.

(68) Buck, R. P. Kinetics of Bulk and Interfacial Ionic Motion: Microscopic Bases and Limits for the Nernst-Planck Equation Applied to Membrane Systems. J. Membr. Sci. 1984, 17, 1-62.

(69) Biesheuvel, P. M. Two-Fluid Model for the Simultaneous Flow of Colloids and Fluids in Porous Media. J. Colloid Interface Sci. 2011, 335, 389-395.

(70) Adamczyk, Z.; Warszynski, P. Role of Electrostatic Interactions in Particle Adsorption. Adv. Colloid Interfac. 1996, 63, 41-149.

(71) Adamczyk, Z.; Belouschek, P.; Lorenz, D. Electrostatic Interactions of Bodies Bearing Thin Double-layers. 1. General Formulation. Ber. Bunsen-Ges. Phys. Chem. 1990, 94, 1483-1492.

(72) Adamczyk, Z.; Belouschek, P.; Lorenz, D. Electrostatic Interactions of Bodies Bearing Thin Double-Layers. 2. Exact Numerical-Solutions. Ber. Bunsen-Ges. Phys. Chem. 1990, 94, 14921499.

(73) Adamczyk, Z.; Belouschek, P.; Lorenz, D. Electrostatic Interactions of Bodies Bearing Thin Double-layers. III. Dissimilar Double-layers. Ber. Bunsen-Ges. Phys. Chem. 1991, 95, 566-573.

(74) Israelachvili, J. N. Intermolecular and Surface Forces, 3rd ed.; Academic Press: San Diego, CA, 2010.

(75) Booth, F. The Dielectric Constant of Water and the Saturation Effect. J. Chem. Phys. 1951, 19, 391-394.

(76) Booth, F. Dielectric Constant of Polar Liquids at High Field Strengths. J. Chem. Phys. 1955, 23, 453-457.

(77) Bazant, M. Z.; Thornton, K.; Ajdari, A. Diffuse-Charge Dynamics in Electrochemical Systems. Phys. Rev. E 2004, 70, 021506.

(78) Goldin, G. M.; Colclasure, A. M.; Wiedemann, A. H.; Kee, R. J. Three-Dimensional Particle-Resolved Models of Li-Ion Batteries to Assist the Evaluation of Empirical Parameters in One-Dimensional Models. Electrochim. Acta 2012, 64, 118-129.

(79) Wiedemann, A. H.; Goldin, G. M.; Barnett, S. A.; Zhu, H.; Kee, R. J. Effects of Three-Dimensional Cathode Microstructure on the Performance of Lithium-Ion Battery Cathodes. Electrochim. Acta 2013, $88,580-588$.

(80) Yeh, H. C.; Yang, R. J.; Luo, W.-J. Analysis of Traveling-Wave Electro-osmotic Pumping with Double-Sided Electrode Arrays. Phys. Rev. E 2011, 83 (1-9), 056326.

(81) Myland, J. C.; Oldham, K. B. Uncompensated Resistance. 1. The Effect of Cell Geometry. Anal. Chem. 2000, 72, 3972-3980.

(82) Masliyah, J. H.; Bhattacharjee, S. Electrokinetic and Colloid Transport Phenomena; John Wiley \& Sons: Hoboken, NJ, 2006.

(83) Sanchez-Gonzalez, J.; Stoeckli, F.; Centeno, T. A. The Role of the Electric Conductivity of Carbons in the Electrochemical Capacitor Performance. J. Electroanal. Chem. 2011, 657, 176-180.

(84) Zhang, L. L.; Zhou, R.; Zhao, X. S. Graphene-Based Materials as Supercapacitor Electrodes. J. Mater. Chem. 2010, 20, 5983-5992.

(85) Cohen, H.; Cooley, J. W. The Numerical Solution of the TimeDependent Nernst-Planck Equations. Biophys. J. 1965, 5, 145-162.

(86) Brumleve, T. R.; Buck, R. P. Numerical Solution of the NernstPlanck and Poisson Equation System with Applications to Membrane Electrochemistry and Solid State Physics. J. Electroanal. Chem. 1978, $90,1-31$

(87) Streeter, I.; Compton, R. G. Numerical Simulation of Potential Step Chronoamperometry at Low Concentrations of Supporting Electrolyte. J. Phys. Chem. C 2008, 112, 13716-13728.

(88) Rubinstein, I.; Zaltzman, B. Extended Space Charge in Concentration Polarization. Adv. Colloid Interface Sci. 2010, 159, 117-129. 